\title{
Tolerance-based strategies for extending the column generation algorithm to the bounded rational dynamic user equilibrium problem
}

\author{
Citation for published version (APA): \\ Wang, D., Liao, F., Gao, Z., \& Timmermans, H. J. P. (2019). Tolerance-based strategies for extending the \\ column generation algorithm to the bounded rational dynamic user equilibrium problem. Transportation \\ Research. Part B: Methodological, 119, 102-121. https://doi.org/10.1016/j.trb.2018.11.008
}

\section{Document license: \\ TAVERNE}

DOI:

10.1016/j.trb.2018.11.008

Document status and date:

Published: 01/01/2019

\section{Document Version:}

Publisher's PDF, also known as Version of Record (includes final page, issue and volume numbers)

\section{Please check the document version of this publication:}

- A submitted manuscript is the version of the article upon submission and before peer-review. There can be important differences between the submitted version and the official published version of record. People interested in the research are advised to contact the author for the final version of the publication, or visit the $\mathrm{DOI}$ to the publisher's website.

- The final author version and the galley proof are versions of the publication after peer review.

- The final published version features the final layout of the paper including the volume, issue and page numbers.

Link to publication

\footnotetext{
General rights

- You may freely distribute the URL identifying the publication in the public portal. follow below link for the End User Agreement:

www.tue.nl/taverne

\section{Take down policy}

If you believe that this document breaches copyright please contact us at:

openaccess@tue.nl

providing details and we will investigate your claim.
}

Copyright and moral rights for the publications made accessible in the public portal are retained by the authors and/or other copyright owners and it is a condition of accessing publications that users recognise and abide by the legal requirements associated with these rights.

- Users may download and print one copy of any publication from the public portal for the purpose of private study or research.

- You may not further distribute the material or use it for any profit-making activity or commercial gain

If the publication is distributed under the terms of Article $25 \mathrm{fa}$ of the Dutch Copyright Act, indicated by the "Taverne" license above, please 


\title{
Tolerance-based strategies for extending the column generation algorithm to the bounded rational dynamic user equilibrium problem
}

\author{
Dong Wang ${ }^{\mathrm{a}, \mathrm{b}}$, Feixiong Liao ${ }^{\mathrm{b}, *}$, Ziyou Gao ${ }^{\mathrm{a}, *}$, Harry Timmermans ${ }^{\mathrm{b}, \mathrm{c}}$ \\ a Institute of Transportation System Science and Engineering, Beijing Jiaotong University, Beijing 100044, China \\ ${ }^{\mathrm{b}}$ Urban Planning Group, Eindhoven University of Technology, Eindhoven $5600 \mathrm{MB}$, The Netherlands \\ ${ }^{\mathrm{c}}$ Department of Air Transportation Management, Nanjing University of Aeronautics and Astronautics, Nanjing 211106, China
}

\section{A R T I C L E I N F O}

\section{Article history:}

Received 17 August 2018

Revised 17 October 2018

Accepted 15 November 2018

\section{Keywords:}

Column generation

Dynamic user equilibrium

Bounded rationality

Temporal resolution

\begin{abstract}
A B S T R A C T
The column generation (CG) algorithm has been widely applied to traffic assignment problems due to its capability of circumventing path enumeration. Incorporating bounded rationality (BR) and dynamics, this paper proposes four tolerance-based strategies for extending the CG algorithm to the bounded rational dynamic user equilibrium model (BR-DUE): (i) a tolerance-based minimum disutility path search strategy is developed to allow travelers seeking satisfactory paths; (ii) a self-adjusted convergence threshold strategy is applied for fast convergence at the intermediate iterations; (iii) a varied temporal resolution scheme, combining exploration and exploitation, is suggested to assign flows to narrow time regions rather than to the whole time horizon; and (iv) a path search skipping strategy is introduced by comparing the lower bound of travel disutility and the minimum disutility between the OD pairs. With these strategies, an efficient tolerancebased column generation (TBCG) algorithm for BR-DUE is developed. Numerical examples are provided to demonstrate that the TBCG algorithm leads to significant computation time reductions without the expense of solution quality, of which the speedup factors are around two compared with using the original CG algorithm.
\end{abstract}

(c) 2018 Elsevier Ltd. All rights reserved.

\section{Introduction}

User equilibrium (UE) traffic assignment has been an active area of transportation research for many years with most model developments motivated by Wardrop's first principle (Wardrop, 1952; Sheffi, 1985; Yang and Bell, 1998; Çolak et al., 2016). Evolved from UE, dynamic user equilibrium (DUE) models (Tong and Wong, 2000; Huang and Lam, 2002; Long et al., 2016) took the time dimension into consideration to enhance the realism of traffic flow propagation. These models assume that travelers have perfect knowledge of traffic conditions throughout the whole network and choose paths with the minimum disutilities. Nevertheless, this assumption is hard to be realized due to the cognitive limitations of travelers. Even if travelers have fully mastered the network information, they would still choose non-optimal paths and/or departure times due to factors such as habit and inertia. It is argued by Simon $(1955,1957)$ that people demonstrate bounded rationality (BR) behavior and seek satisfactory rather optimal solutions because of limited information and limited capability of pro-

\footnotetext{
* Corresponding authors.

E-mail addresses: F.Liao@tue.nl (F. Liao), zygao@bjtu.edu.cn (Z. Gao).
} 


\section{Notations}

$G(N, A)$ the traffic network composed of node set $N$ and link set $A$

$R S$ the set of OD pairs

a a link of $G, a \in A$

rs an OD pair, $r s \in R S$

$k \quad$ a time interval, $k \in K$

$p, \bar{p} \quad$ two paths

Prs the set of paths of $r s$

$P_{n}^{r s} \quad$ the set of paths of $r s$ at iteration $n$

$Q^{r s} \quad$ the demand of $r s$

$\Delta \quad$ the minimum length of a time interval

$K$ the set of time intervals corresponding to $\Delta$

$\Delta_{n} \quad$ the length of a time interval at iteration $n$

$K_{n} \quad$ the set of time intervals corresponding to $\Delta_{n}$

$\delta^{r s} \quad$ the half-length of the arrival time window without penalty for travelers of OD pair $r s$

$k^{\text {rs* }} \quad$ the preferred arrival time of $r s$

$\mu, \omega \quad$ the scale parameters, $\mu, \omega \in(0,1)$

$\alpha \quad$ the unit disutility of travel time

$\beta \quad$ the unit disutility of early arrival time

$\gamma \quad$ the unit disutility of late arrival time

$\varepsilon^{r s} \quad$ the relative indifference threshold of travelers of $r s$ toward path switch

$\varepsilon \quad$ convergence threshold

$\varepsilon^{r s} \quad$ the minimum relative convergence threshold of $r s$

$\varepsilon_{n}^{r s} \quad$ the relative convergence threshold of $r s$ at iteration $n$

$\varepsilon_{\max }^{r s} \quad$ the maximum relative convergence threshold of $r s$

$f_{p}^{r s}(k) \quad$ the flow that enters path $p$ of $r s$ during $k$

$f \quad$ the vector of $f_{p}^{r s}(k)$

$f_{n} \quad$ the vector of $f_{p}^{r s}(k)$ at iteration $n$

$c_{a} \quad$ the capacity of link $a$

$t_{a}^{0} \quad$ the free flow travel time of link $a$

$t_{a}(k, f) \quad$ the travel time of link $a$ for travelers entering at interval $k$

$t_{p}^{r s}(k, f)$ the travel time of path $p$ incurred by travelers departing with traffic flow vector $f$ during time interval $k$ for OD pair $r s$

$c_{p}^{r s}(k, f)$ the path disutility of $p$ incurred by travelers departing with $f$ during $k$ for $r s$

$c_{\min }^{r s}(f)$ the minimum disutility of $r s$

$c^{r s}(k, 0) \quad$ the minimum disutility of paths with zero flow of $r s$ at $k$

$\Phi_{n}^{r s} \quad$ The potential time-dependent path set (PTPS) at iteration $n$ of $r s$

$|S E T| \quad$ the number of elements in an ordinary set SET

cessing information. In response, the BR of travelers or consumers has been incorporated in a number of traffic assignment models (Di and Liu, 2016).

Incorporating departure time choice, Szeto and Lo (2006) introduced the tolerance-based DUE problem and discussed its solution characteristics. Based on this work, Han et al. (2015) developed a bounded rational dynamic user equilibrium (BRDUE) model with variable tolerances, of which the DUE and tolerance-based DUE are special cases. Solution existence and three computational algorithms were proposed based on the corresponding variational inequalities (VI) and differential VI formulations. BR has also been incorporated into process models to capture the day-to-day learning behavior and traffic flow dynamics (Guo and Liu, 2011; Wu et al., 2013). Recently, Di and Liu (2016) provided a comprehensive review of theoretical models and empirical evidence of route choice with BR, and confirmed that travelers do not usually choose the paths of the shortest travel times or the lowest disutilities. Despite the appealing theoretical developments, the numerical studies of the few existing BR-DUE models were performed on pre-defined path sets. For example, Szeto and Lo (2006) considered 6 paths in a 10-node network and pointed out the necessity of path generation as a future research direction. Han et al. (2015) selected 119 paths of the Sioux Falls (24-node) network to assess the suggested algorithms to the BR-DUE model. Path enumeration may be possible for some transport networks of special topologies. However, the number of possible time-dependent paths may be too large even for small general networks, and path enumeration is almost impossible for larger networks. Therefore, a method of path generation rather than enumeration is needed.

For solving static traffic assignment problems, Leventhal et al. (1973) developed the seminal column generation (CG) algorithm of path generation in general transport networks. This algorithmic scheme has been widely used in recent studies 
including UE (e.g., Di Lorenzo et al., 2014), DUE (Zhou et al., 2008; Lu et al., 2009; Long et al., 2013b; Zhou and Taylor, 2014; Levin et al., 2015), and other traffic assignment model variants (e.g., Szeto et al., 2013; Zhang et al., 2013; Ryu et al., 2016); however, to the best of our knowledge, no application has hitherto been devoted to BR-DUE.

Path search and network loading are the two most time-intensive components in the CG algorithm for traffic assignments. Several strategies for balancing these two components have been proposed. Panicucci et al. (2007) suggested performing a column generation procedure (path search) after a certain number of iterations rather than at every iteration. It is found that the best value of the iteration gap varies between 8 and 12 . Aligned with this effort, Di Lorenzo et al. (2014) proved that the column generation procedure must be applied within a prefixed number of iterations to guarantee convergence. The results of computational experiments illustrated that their proposed algorithm outperformed other algorithms. Based on this work, Galligari and Sciandrone (2017) designed a strategy adjusting the prefixed number for each OD pair. This strategy yielded substantial computational time-savings, whilst retaining the global convergence property. However, these strategies are only limited to the spatial paths.

Considering both BR and the temporal dimension, this study develops a tolerance-based column generation (TBCG) algorithm to solve the BR-DUE problem. Four tolerance-based strategies extending the CG algorithm are proposed across the spatial and temporal dimensions. First, a tolerance-based minimum disutility path search strategy is proposed to seek satisfactory paths. Second, a self-adjusted convergence threshold strategy is adopted to perform pseudo-equilibrium assignments at the intermediate iterations. Third, a varied temporal resolution scheme combing temporal exploration and exploitation is designed to assign flows to narrow time regions rather than to the whole time horizon. Lastly, a path search skipping strategy is developed to perform path searches when necessary. Using these spatial and temporal strategies, the TBCG algorithm efficiently finds the BR-DUE solutions.

The remainder of this paper is organized as follows. Section 2 provides the preliminary knowledge about path disutility, and the DUE and BR-DUE conditions. Section 3 introduces the tolerance-based strategies for extending the CG algorithm to solve the BR-DUE problem. Numerical examples are given in Section 4 to assess the effectiveness of the proposed TBCG algorithm. Finally, conclusions and recommendations for future work are provided.

\section{Preliminaries}

This section provides some preliminary knowledge about the DUE and BR-DUE problems, including the definitions of path disutility function, DUE and BR-DUE conditions, and the traditional CG algorithm.

\subsection{Path disutility}

As a primary factor of trips, travel time has been the focus of attention in several appealing properties such as Lipschitz continuity, monotonicity, and differentiability with respect to traffic flow. Path travel time has been widely used as the subject of minimization in various DUE models (Lo and Szeto, 2002; Lu et al., 2013; Long et al., 2016). Formally, a path travel time can be calculated using the following nested function

$$
t_{p}^{r s}(k, f)=t_{a_{1}}(k, f)+t_{a_{2}}\left(k+t_{a_{1}}, f\right)+\cdots+t_{a_{m}}\left(k+t_{a_{1}}+\ldots+t_{a_{m-1}}, f\right)
$$

where $k$ denotes a time interval, $f$ is the vector of path flow $f_{p}^{r s}(k)$ entering path $p$ of OD pair $r s$ during $k, t_{p}^{r s}(k, f)$ is the path travel time of $p$ incurred by travelers departing during $k$ for $r s . t_{a}(k, f)$ is the travel time on link $a$ when entering at interval $k$. We simplify the notations: $t_{a_{1}}=t_{a_{1}}(k, f), t_{a_{2}}=t_{a_{2}}\left(k+t_{a_{1}}, f\right), \ldots$, for short. $a_{1}, a_{2}, \ldots, a_{m}$ are consecutive links of path $p=a_{1}-a_{2}-\ldots-a_{m}$. The link travel time can either be obtained by a link travel time function (Nie and Zhang, 2005) or determined through different dynamic network loading methods, such as cell transmission model (CTM) (Daganzo, 1995), link transmission model (LTM) (Yperman, 2007; Long et al., 2013b), and point queue model (Huang and Lam, 2002).

Besides travel time, monetary cost and penalties of unpunctual trips are important considerations for trip scheduling. Disutility or generalized travel cost is defined directly from the outcomes such as travel time and being early or late (Fosgerau et al., 2010). Travelers hold preferred arrival times (PAT) and incur early or late arrival penalties when the actual arrival times are less than or exceeding their preferred arrival times. Incorporating the unpunctual penalties, the path disutility is calculated by a piecewise weighted sum as

$$
c_{p}^{r s}(k, f)=\alpha t_{p}^{r s}(k, f)+ \begin{cases}\beta\left[k^{r s *}-\delta^{r s}-k-t_{p}^{r s}(k, f)\right] & \text { if } k+t_{p}^{r s}(k, f)<k^{r s *}-\delta^{r s} \\ \gamma\left[k+t_{p}^{r s}(k, f)-k^{r s *}-\delta^{r s}\right] & \text { if } k+t_{p}^{r s}(k, f)>k^{r s *}+\delta^{r s} \\ 0 & \text { otherwise }\end{cases}
$$

where $c_{p}^{r s}(k, f)$ denotes the path disutility of $p$ incurred by travelers departing during $k$ for $r s ; \alpha, \beta$ and $\gamma$ are the unit costs of travel time, early and late arrival time respectively. $k^{r s *}$ is the preferred arrival time of travelers for $r s ;\left[k^{r s *}-\delta^{r s}, k^{r s *}+\delta^{r s}\right]$ is the indifferent band of arrival times without unpunctual penalties. Although this piecewise linear disutility function is not fully realistic but widely adopted in DUE models (Huang and Lam, 2002; Szeto and Lo, 2004; Han et al., 2011). 


\subsection{DUE conditions}

Dynamic traffic assignment (DTA) is an extension of the static traffic assignment (STA) problem that aims to capture dynamic traffic flows and serves traffic operational management in the short-term. The DUE condition is stated as follows: for each OD pair at each time interval, the path disutilities experienced by travelers departing at the same time are equal and minimal. This condition is a dynamic version of Wardrop's first principle and implies that, at DUE, only those timedependent paths between any OD pairs that have the minimal disutilities are used, and those paths that are not used must have disutilities higher than or equal to the minimal disutilities; in addition, no individual can reduce his/her path disutility by unilaterally adapting the departure time and path. Formally, it can be expressed as

$$
c_{p}^{r s}\left(k, f^{*}\right)\left\{\begin{array}{l}
=c_{\min }^{r s}\left(f^{*}\right) \text { if } f_{p}^{r s *}(k)>0 \\
\geq c_{\min }^{r s}\left(f^{*}\right) \text { if } f_{p}^{r s *}(k)=0
\end{array} \forall p \in P^{r s}, r s \in R S, k \in K\right.
$$

where $c_{\min }^{r s}(f)$ is the minimum disutility of $r s ; K$ and $R S$ are the sets of all time intervals and OD pairs respectively, and $P^{r s}$ is the set of paths of $r$.

The DUE problem is to find $f^{*}$ such that Eq. (3) and the following demand and non-negativity constraints are satisfied.

$$
\begin{aligned}
& \sum_{p \in P^{r s}} \sum_{k \in K} f_{p}^{r s}(k)=Q^{r s} \forall r s \in R S \\
& f_{p}^{r s}(k) \geq 0 \forall p \in P^{r s}, r s \in R S, k \in K
\end{aligned}
$$

where $Q^{r s}$ is the demand of $r s$.

The existence and uniqueness of the solution for DUE depend on the relationship between the path disutility functions and the path flows. When the path disutility functions are continuous with path flows, the DUE problem has at least one solution, and this solution is unique when the Jacobian matrix of the path disutility functions is positive definite.

To obtain solutions for DUE, a number of traffic assignment algorithms were proposed. For example, Lu et al. (2009) proposed a descent direction method and proved this method outperforms the method of successive average (MSA). Carey and $\mathrm{Ge}$ (2012) compared four algorithms and concluded that the route-swapping method (Smith and Wisten, 1995) and the simple projection method (Rubio-Ardanaz et al., 2003) converge faster than the MSA and the alternating direction method (Lo and Szeto, 2002). Long et al. (2013b) applied the extra-gradient method to the DUE problem. The convergence properties of these algorithms are derived from different properties of the path disutility functions such as continuity, pseudomonotonicity, and co-coercivity.

\subsection{BR-DUE conditions}

As aforementioned, BR-DUE models (Han et al., 2015) have attempted incorporating BR reflecting that travelers do not necessarily choose paths of the minimum disutilities. The condition of BR-DUE is stated as: for each OD pair at each time interval, the disutilities experienced by travelers departing at the same time are no larger than the minimum value plus a threshold. Formally, it can be expressed as

$$
c_{p}^{r s}\left(k, f^{*}\right) \in\left[c_{\min }^{r s}\left(f^{*}\right), c_{\min }^{r s}\left(f^{*}\right) \cdot\left(1+\varepsilon^{r s}\right)\right] \text { if } f_{p}^{r s *}(k)>0 \forall p \in P^{r s}, r s \in R S, k \in K
$$

where $\varepsilon^{r s}$ is the threshold of acceptable relative differences in the travel disutilities experienced by travelers of OD pair rs. It is obvious that Eq. (6) is equivalent to Eq. (3) when $\varepsilon^{r s}$ equals zero, which means that the BR-DUE is a more general form. The BR-DUE problem can be formulated as a finite-dimensional variational inequality problem $\operatorname{VI}(f, \Omega)(\mathrm{Han}$ et al., 2015$)$ to find a vector $f^{*}$ such that

$$
\begin{aligned}
& \sum_{r s \in R S} \sum_{p \in P^{r s}} \sum_{k \in K} \tilde{c}_{p}^{r s}\left(k, f^{*}\right)\left[f_{p}^{r s}(k)-f_{p}^{r s *}(k)\right] \geq 0 \forall f \in \Omega \\
& \Omega=\left\{f \mid f \geq 0, \sum_{p \in P^{r s}} \sum_{k \in K} \Delta \cdot f_{p}^{r s}(k)=Q^{r s}, \forall r s \in R S\right\}
\end{aligned}
$$

where $\Delta$ is the minimum length of a time interval and $\tilde{c}_{p}^{r s}\left(k, f^{*}\right)$ is formulated as follows

$$
\tilde{c}_{p}^{r s}\left(k, f^{*}\right)=\max \left\{c_{p}^{r s}\left(k, f^{*}\right), c_{\min }^{r s}\left(f^{*}\right) \cdot\left(1+\varepsilon^{r s}\right)\right\}
$$

Han et al. (2015) pointed out that the existence of BR-DUE requires a mapping operator, which is a more general form of path disutility functions, to be continuous. This condition is weaker than the counterpart of DUE and the solutions are not unique. For the discrete time $\operatorname{VI}(f, \Omega)$ problem Eqs. (7)-(9), the analyses of the existence and non-uniqueness of the solutions are presented in Appendix A. 


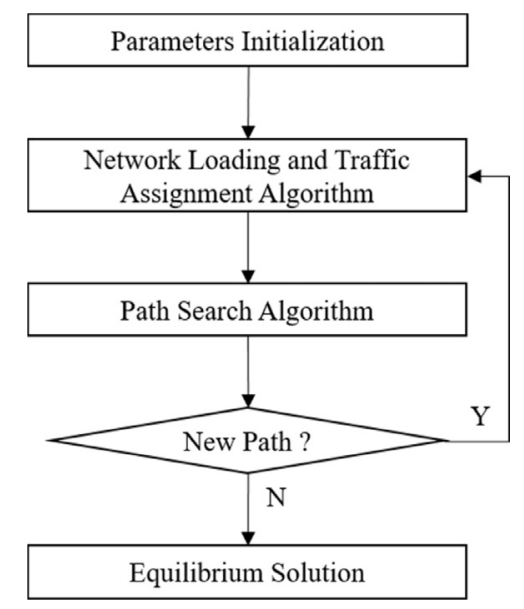

Fig. 1. Flowchart of the traditional CG algorithm.

\subsection{Column generation algorithm}

To circumvent path enumeration, the CG algorithm has been embedded into the solution algorithms for the STA and DTA problems. Specifically, at the initialization stage, a non-empty path set for each OD pair is created by the minimum disutility path search algorithms. Traffic assignment is performed on the path sets to create a snapshot of traffic flow realization, upon which new minimum disutility paths will be found and evaluated if being added to the path sets. This process is repeated until no more new path can be found (Chen et al., 2001; Lu et al., 2009, 2016). The existing CG algorithms for STA and DTA have a similar skeleton as shown in Fig. 1. The CG algorithms for the DTA problem requires a higher magnitude of run-time complexities, which are $O(|N| \cdot|A| \cdot|K|)$ to conduct path searches and $O\left(\sum_{r s}\left|P^{r s}\right| \cdot\left|A^{\prime}\right| \cdot|K| \cdot m\right)$ to load path flows at one iteration, where $\left|A^{\prime}\right|$ is the maximum number of links in a path, $m$ is the number of dynamic network loadings, $|N|$, $|A|$, $|K|$ and $\left|P^{r s}\right|$ denote the numbers of elements in the corresponding sets. Despite effective, the majority CG algorithms for STA and DTA have followed a rigid structure and thus can be further improved by introducing self-adjustment in the process.

\section{Tolerance-based column generation for BR-DUE model}

This section presents four strategies covering both the spatial and temporal dimensions to extend the CG algorithm to solve BR-DUE (Eq. (6)). Thereafter, we propose the tolerance-based column generation (TBCG) algorithm and present the algorithmic flowchart and pseudo-code. In this paper, the point queue method (Huang and Lam, 2002; Zhou and Taylor, 2014 ) is applied for modeling traffic flow propagation. To illustrate the mechanisms of the TBCG algorithm, a small case is used for detailing the key descriptions. To keep consistency, the notations used above attached with $n$ refer to the same entities at iteration $n$. Furthermore, a potential time-dependent path set (PTPS) is created to store certain path and departure time pairs having potentials to be the BR-DUE solutions. The PTPS at iteration $n$ of $r$ is denoted by $\Phi_{n}^{r s}=\{(p, k)\}$.

\subsection{Tolerance-based strategies}

As path search and network loading constitute the most time-intensive components in a CG algorithm for traffic assignment, improving and balancing these two components are crucial for accelerating the algorithm. When considering the temporal dimension, time discretization is needed to solve the DTA problems in the discrete domain, since there is no known method for solving complex time-continuous models analytically. As indicated in Huang and Lam (2002), the length of one unit of time interval should be set small enough so that the discrete DTA model approximates its continuous counterpart. As travelers choose departure times and paths simultaneously, a smaller time interval leads to a large multiplier increase of the choices compared to the static UE, which aggravates the burden of computation. In addition, to solve BR-DUE, BR should be included in the path search and network loading components. To address these issues, the following strategies are suggested, particularly to be applied at the intermediate iterations

(i) a tolerance-based minimum disutility path search (TBMDPS) strategy is applied to allow for BR and reduce the number of path flow variables;

(ii) self-adjusted convergence thresholds are used to ensure fast convergences;

(iii) for temporal exploration, the temporal resolution is set stationary to a explore the potential time region of BR-DUE; regarding temporal exploitation, the temporal resolution is dynamically set high to meet the required convergence precision for BR-DUE;

(iv) a path search skipping strategy is adopted to decrease the number of path searches. 


\subsubsection{Tolerance-based minimum disutility path search}

In the dynamic context, time-dependent paths with the minimum disutility can be found by dynamic programming (Dean, 2004; Liao, 2017). When the newly generated path $\bar{p}$ with disutility $c_{\bar{p}}^{r s}\left(k, f_{n}\right)$ satisfying Eq. (10) does not belong to the path set $P_{n}^{r s}$ of $r s$ at iteration $n, \bar{p}$ is added to $P_{n}^{r s}$. A similar criterion is widely used in the CG algorithm for the DUE problem (Lu et al., 2009, 2016).

$$
c_{\min }^{r s}\left(f_{n}\right)-c_{\bar{p}}^{r s}\left(k, f_{n}\right) \geq 0
$$

In contrast to adding a new path with the minimum disutility to the path set, strategy $(i)$ compares $c_{\tilde{p}}^{\text {rs }}\left(k, f_{n}\right)$ and the minimum disutility of paths in $P_{n}^{r s}$. Only path $\bar{p}$ with extra disutility losses larger than a threshold is added to the path set, as shown in Eq. (11), where $\varepsilon^{r s}$ is a relative indifference threshold of travelers of OD pair $r$ toward path switch. This condition is consistent with the travel behavior of BR. Specifically, in a long-term equilibrium process, travelers depart from their origins at preferred time intervals and travel on preferred paths. Despite the existence of paths with similar or even smaller disutilities, they may prefer the familiar paths due to the inertial to adapt. The travelers switch paths only if the new ones lead to extra less disutilities. Note that strategy $(i)$ is OD related and will degenerate into Eq. (10) if $\varepsilon^{\text {rs }}$ is equal to zero.

$$
c_{\min }^{r s}\left(f_{n}\right)-c_{\tilde{p}}^{r s}\left(k, f_{n}\right) \geq \varepsilon^{r s} \cdot c_{\min }^{r s}\left(f_{n}\right)
$$

Let $\bar{C}_{i}(k)$ denotes the minimum disutility of arriving at node $i$ and time interval $k, \bar{t}_{i j}^{n}(k)$ the arrival time at $j$ and $\bar{c}_{i j}^{n}(k)$ the disutility of traversing link $(i, j)$ when departing from $i$ during $k$ at iteration $n, F_{j}^{i}\left(\bar{t}_{i j}^{n}(k)\right)$ is a two-tuple vector recording the preceding link and interval. The pseudo-code of the TBMDPS at iteration $n$ is presented in Algorithm 1, which delivers the best worst-case run-time complexity.

Remark 1. Strategy (i) accelerates the CG algorithm by decreasing the size of the path sets. The size of the path sets in DTA models plays a major role in the computation time due to the large path expansion factor accounting for time-dependency. Specifically, the size of the time-dependent paths of DTA is $\sum_{r s}\left|P^{r s}\right| \cdot|K|$ (Huang and Lam, 2002; Long et al., 2016). This value is decreased to $\sum_{r s}\left(\left|P^{r s}\right|-l\right) \cdot|K|$ if $l$ paths are discarded due to the TBMDPS. The larger the value of $|K|$, the larger decrease it incurs. The path $\bar{p}$ satisfying Eq. (11), generated by the TBMDPS, is called an acceptable path. The input parameters of this algorithm are flexible to be linked with iteration-related variables, which make it possible to combine with the temporal strategies.

\subsubsection{Self-adjusted convergence thresholds}

Convergence curves depicted by the gap functions of DUE models usually become flat when the solutions approach the equilibrium (Lo and Szeto, 2002; Long et al., 2013b). Using a fixed small convergence threshold would lead to long computation times at the intermediate iterations. To allow for BR and drive the intermediate steps fast, strategy (ii) concerns the self-adjusted convergence thresholds, formulated in Eq. (12).

$$
c_{p}^{r s}\left(k, f_{n}\right)-c_{\min }^{r s}\left(f_{n}\right) \leq \varepsilon_{n}^{r s} \cdot c_{\min }^{r s}\left(f_{n}\right)
$$

where $\varepsilon_{n}^{r s}$ is the relative convergence threshold of travelers between OD pair $r s$ at iteration $n$ toward time change.

For each OD pair rs, Eq. (12) has a similar form to the BR-DUE condition, but the difference lies in the fluctuating convergence threshold $\varepsilon_{n}^{\text {rs }}$ with reference to iteration $n$. Based on the relationship between the acceptable path $\bar{p}$ generated from strategy $(i)$ and $P_{n}^{r s}, \varepsilon_{n}^{r s}$ is changed differently in the following two cases.

Case (1): At least one acceptable path does not belong to the current path set, i.e. $\exists \bar{p} \notin P_{n}^{r s}$.

In this case, some traffic flows are more likely to shift to the new paths. To ensure fast convergence, the value of the convergence threshold increases by the following formula

$$
\varepsilon_{n+1}^{r s}=\min \left(\frac{1}{\mu} \cdot \varepsilon_{n}^{r s}, \varepsilon_{\max }^{r s}\right)
$$

where $\mu \in(0,1)$ is a parameter for scaling and $\varepsilon_{\max }^{r s}$ is the maximum convergence tolerance for $r s$. Eq. (13) uses $\varepsilon_{\max }^{r s}$ as the upper bound to prevent the unrestricted increase of $\varepsilon_{n+1}^{r s}$.

Case (2): All acceptable paths fall within the current path set, i.e. $\forall \bar{p} \in P_{n}^{r s}$.

In this case, the traffic flows have converged to a state, in which all used paths belong to $P_{n}^{r s}$ with $\varepsilon_{n}^{r s}$. To obtain a more precise assignment, $\varepsilon_{n+1}^{r s}$ is decreased as below

$$
\varepsilon_{n+1}^{r s}=\max \left(\mu \cdot \varepsilon_{n}^{r s}, \varepsilon^{r s}\right)
$$

where $\varepsilon^{r s}$ is used as a lower bound to ensure a required tolerance.

Remark 2. Strategy (ii) accelerates the CG algorithm by decreasing the number of network loadings. A larger $\varepsilon_{n}^{r s}$ ensures faster convergence at iteration $n$. When no new path is generated, $\varepsilon_{n}^{r s}$ is decreased until a required convergence threshold to obtain a BR-DUE solution. 


\subsubsection{Temporal exploration and exploitation}

The challenge in DTA mainly stems from the path extension in the temporal dimension. The previous CG algorithms for DTA assign flows to the generated paths across the whole time horizon with a fixed time resolution. This strategy concerns the varied time resolutions for temporal exploration and exploitation.

Temporal exploration

Suppose that an acceptable path $\bar{p}$ is found by strategy $(i)$ at iteration $n$. It may be the case that not all time intervals for $\bar{p}$ are potential for the traffic assignment, especially when the time intervals fall at the beginning or the end of the time horizon. The temporal exploration is adopted to find the potential time intervals and add the corresponding time-dependent paths to PTPS. Specifically, given path $p \in P_{n}^{r s}$, if there are time-dependent paths $(p, k)$ for $\forall k \in K$ satisfying Eq. (12) and falling out of $\Phi_{n}^{r s}$, add $(p, k)$ to $\Phi_{n}^{r s}$ and assign the flows to them. Usually, the neighboring time intervals of $k$ are likely to be potential. This process is conducted until no new time-dependent path satisfies Eq. (12).

Temporal exploitation

A higher temporal resolution of DTA means that the traffic flows are propagated more accurately. The side-effects of high temporal resolution are the increase in the size of time-dependent paths and the need of a long flow propagation process, and thus dramatically more computation time is required. It is well known that the traffic flows are assigned to a small proportion of continuous time regions rather the full time horizon in transport networks with bottlenecks. Based on this notion, the TBCG algorithm starts from a low temporal resolution to ensure converging to a satisfactory equilibrium result at a considerable speed, and ends up with a high temporal resolution to meet the required criterion.

We denote the temporal resolution at iteration $n$ by $\Delta_{n}$, and $\Delta$ is used as a lower bound of $\Delta_{n}$. Whether to decrease $\Delta_{n}$ or not depends on the relationship between the acceptable path $\bar{p}$ and $P_{n}^{r s}$, which is similar to the two cases above. In Case (1), we keep $\Delta_{n}$ the same as the last iteration. When falling within Case (2), temporal exploitation is adopted before decreasing $\varepsilon_{n}^{r s}$. If $\Delta_{n}>\Delta$, temporal exploitation is trigged by the following formula

$$
\Delta_{n+1}=\max \left(\omega \cdot \Delta_{n}, \Delta\right)
$$

where $\omega \in(0,1)$ is a resolution parameter, - is an integer-floor operator. Besides transforming the link attributes (e.g., link capacity and free flow travel time) in relation to $\Delta_{n+1}$, each time-dependent path $(p, k)$ in $\Phi_{n}^{r s}$ is replaced by $\left(p, k^{\prime}\right)$ for $\forall k^{\prime}$ satisfying $k^{\prime}$ w. $\Delta_{n+1} \in[k, k+1) \cdot \Delta_{n}$ and the corresponding flow $f_{p}^{r s}(k)$ are divided into $\Delta_{n} / \Delta_{n+1}$ parts equally and spread to each $\left(p, k^{\prime}\right)$. If $\Delta_{n}=\Delta$, the spatial exploitation is performed by decreasing $\varepsilon_{n}^{\text {rs }}$ as shown in Eq. (14).

Regarding temporal exploration, it is a process of extending the time dimension of PTPS from several time intervals. Comparatively, strategy (i) can be regarded as spatial exploration, which is a process to add new acceptable paths to $P_{n}^{r s}$ for temporal exploration. Traffic flows are assigned based on the updated PTPS and the unused paths are removed from $P_{n}^{r s}$. Although the path set $P_{n}^{r s}$ may be contracted after one time of traffic assignment, the removed time-dependent paths have higher disutilities, which guarantees the occurrence of Case (2).

Remark 3. Strategy (iii) is performed from a low temporal resolution, which accelerates the original CG algorithm by decreasing the size of the path set. For example, when the resolution at iteration $n$ is twice the required one, i.e., $\Delta_{n}=2 \cdot \Delta$, half the time-dependent paths may be used to assign traffic flows. Among them, only the time-dependent paths satisfying Eq. (12) are added to PTPS according to the temporal exploration. The exploitation procedure leads to the decrease of $\Delta_{n}$ and $\varepsilon_{n}^{r s}$, which guarantees that the TBCG algorithm converges to a state of BR-DUE with the required temporal resolution and convergence tolerance.

\subsubsection{Minimum disutility path search skipping}

This strategy suggests that the TBCG algorithm performs the TBMDPS only at the time intervals that may generate acceptable paths. Whereas, the classical CG algorithm for DTA (Lu et al., 2009) finds the path with the minimum disutility at every departure time interval at every iteration. Note that the minimum disutility of paths with zero flow of $r s$ at $k$, denoted as $c^{r s}(k, 0)$, is the lower bound at $k$ for $r s$. Whether or not to allocate traffic flows to interval $k$ at iteration $n$ depends on the relationship between $c^{r s}(k, 0)$ and the minimum disutility of $r s, c_{\min }^{r s}\left(f_{n}\right)$. If $c^{r s}(k, 0)<c_{\min }^{r s}\left(f_{n}\right)$, interval $k$ is a potential candidate and path search is performed. In contrast, it is impossible to assign traffic flows to the paths at $k$ if $c^{r s}(k, 0) \geq c_{\min }^{r s}\left(f_{n}\right) \cdot\left(1+\varepsilon_{n}^{r s}\right)$. In other words, interval $k$ satisfying Eq. (16) means that the traffic flows are more likely to concentrate on the existing time-dependent paths. Hence, there is no need for path search at this interval at iteration $n$. The remaining intervals with $c^{r s}(k, 0)$ falling between $c_{\min }^{r s}\left(f_{n}\right)$ and $c_{\min }^{r s}\left(f_{n}\right) \cdot\left(1+\varepsilon_{n}^{r s}\right)$ may be assigned with traffic flows and path searches are conducted.

$$
c_{\min }^{r s}\left(f_{n}\right) \cdot\left(1+\varepsilon_{n}^{r s}\right)<c^{r s}(k, 0)
$$

where 0 is a zero flow vector.

Remark 4. Strategy (iv) accelerates the CG algorithm by decreasing the number of path searches. For the traditional CG algorithm, $|K|$ times of path searches are needed to find the potential paths. This value will decrease to $\left|K_{n}\right|-\kappa$ when $\kappa$ intervals satisfy Eq. (16), where $K_{n}$ is the set of time intervals corresponding to $\Delta_{n}$.

Table 1 compares the CG algorithms for UE, DUE and BR-DUE models in terms of path search and network loading. The "cost" refers to generalized travel cost, which may be linked to one or multiple factors. As presented, the temporal 
Table 1

Comparison of the CG algorithms for different UE models.

\begin{tabular}{|c|c|c|c|c|c|c|c|}
\hline & \multirow[t]{2}{*}{ Reference } & \multicolumn{2}{|l|}{ Path search } & \multicolumn{3}{|c|}{ Network loading } & \multirow[t]{2}{*}{$\begin{array}{l}\text { Temporal } \\
\text { resolution }\end{array}$} \\
\hline & & Search method & $\begin{array}{l}\text { Search } \\
\text { frequency per } \\
\text { iteration }\end{array}$ & $\begin{array}{l}\text { Loading } \\
\text { method }\end{array}$ & $\begin{array}{l}\text { Convergence } \\
\text { threshold }\end{array}$ & Accuracy & \\
\hline UE & $\begin{array}{l}\text { Chen et al. } \\
\text { (2011), } \\
\text { Ryu et al. } \\
\text { (2016). }\end{array}$ & $\begin{array}{l}\text { minimum cost } \\
\text { path search }\end{array}$ & once & static & fixed & $\varepsilon$ & - \\
\hline DUE & $\begin{array}{l}\text { Zhou et al. } \\
\text { (2008), Lu et al. } \\
\text { (2013). }\end{array}$ & $\begin{array}{l}\text { minimum cost } \\
\text { path search }\end{array}$ & $\begin{array}{l}\text { for each time } \\
\text { interval }\end{array}$ & dynamic & fixed & $\Delta$ and $\varepsilon$ & fixed \\
\hline BR-DUE & current study & TBMDPS & $\begin{array}{l}\text { for intervals } \\
\text { dis-satisfying } \\
\text { Eq. (16) }\end{array}$ & dynamic & self-adjusted & $\Delta$ and $\varepsilon^{r s}$ & varied \\
\hline
\end{tabular}

resolution is listed after both path search and network loading as it is implicated in both processes. Compared with the CG algorithm for UE and DUE, the TBCG algorithm for BR-DUE has merits of self-adjustment without sacrificing accuracy at the final iteration. Regarding the spatial accuracy, the OD-related parameter $\varepsilon^{r s}$ has the ability to reflect the heterogeneity of trips, while $\varepsilon$ in this table is a convergence threshold for UE and DUE. When $\varepsilon^{r s} \cdot c_{\min }^{r s}\left(f_{n}\right)$ equals $\varepsilon$, TBCG algorithm has the same spatial accuracy as the CG algorithms for UE and DUE.

\subsection{Tolerance-based column generation algorithm}

The above four strategies extend the original CG algorithm from different aspects. Considering the run-time of the TBCG algorithm, time complexity analysis is described simply as follows. When the recursive formulations (Dean, 2004) are used to search for the tolerance-based minimum disutility paths, the run-time complexity is $O(|N| \cdot|A| \cdot|K|)$ at one iteration. The path search skipping strategy and temporal resolution scheme are developed to decrease the value of $|K|$. The dynamic network loading phase consumes $O\left(\sum_{r s}\left|P^{r s}\right| \cdot\left|A^{\prime}\right| \cdot|K| \cdot m\right)$ run-time in the worst case to load path flows. The TBMDPS strategy decreases $\sum_{r S}\left|P^{r s}\right|$, the fluctuating convergence threshold strategy decreases $m$, and the temporal resolution scheme decreases $|K|$. Conversely, the number of iterations may increase due to the changing temporal resolution. Thanks to the strategy of self-adjusted convergence thresholds, the TBCG algorithm can converge to an equilibrium state with less network loadings overall.

Accordingly, the TBCG algorithm has three loops as depicted in Fig. 2. As shown within the blue box, the outer loop is for updating $P_{n}^{r s}$, enhancing $\Delta_{n}$ or decreasing $\varepsilon_{n}^{r s}$. When new paths $p$ are generated at iteration $n$, which means that some flows will shift to these paths, a large $\varepsilon_{n}^{r s}$ ensures fast convergence. When no new path is generated and the result at the current iteration has converged at a low temporal resolution, the temporal exploitation procedure is performed to enhance $\Delta_{n}$. Whether or not to exit the outer loop also depends on $\varepsilon_{n}^{r s}$. If $\varepsilon_{n}^{r s}=\varepsilon^{r s}$, the algorithm has converged to a BR-DUE state with the required convergence tolerance at the highest temporal resolution; then, the outer loop is exited. Otherwise, traffic flows are assigned repeatedly by decreasing $\varepsilon_{n}^{r s}$. The inner loop denotes the temporal exploration procedure. Given a path set, all potential time intervals are added by alternately performing traffic assignment and temporal exploration. Besides, another loop is implicit in the traffic assignment algorithm to obtain a path flow pattern. It is noteworthy that the CG algorithms generally converge in small numbers of outer iterations and do not enumerate all paths.

The proposed four strategies significantly revise the CG algorithm for speeding-up but do not modify the property of convergence. That is, if the convergence conditions of the original CG algorithm are satisfied, the TBCG algorithm can also converge to a BR-DUE state. This conclusion is presented as follows.

Theorem 1. The tolerance-based strategies in the TBCG algorithm maintain the convergence property of the CG algorithm.

Proof. See Appendix B.

Incorporating the proportional swap system (Smith, 1984; Guo et al., 2017) for traffic assignment, we conclude the convergence of the TBCG algorithm as Corollary 1.

Corollary 1. The TBCG algorithm is convergent if the path disutility is a monotone function of path flows.

Proof. See Appendix C.

Corollary 1 states the convergence of the TBCG algorithm under the monotonicity assumption. Besides the proportional swap system, other algorithms and convergence conditions can be embedded into the TBCG algorithm. For example, the convergence conditions of the self-adaptive projection method and proximal point method (Han et al., 2015) rely on the pseudomonotonicity and semi-strictly quasi-monotonicity respectively. However, the path disutility is in general not a monotone 


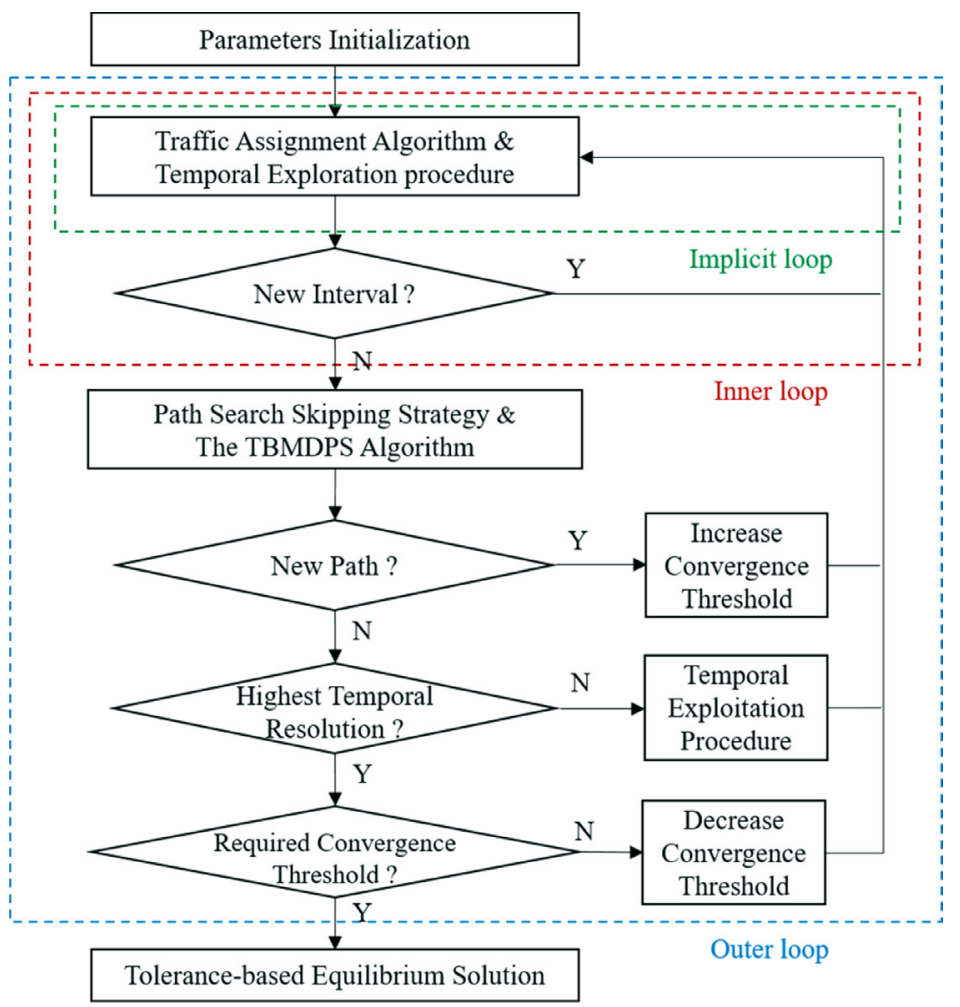

Fig. 2. Flowchart of the TBCG algorithm.

function with respect to the path flow (Huang and Lam, 2002). Compared with other algorithms for traffic assignment, the route-swapping algorithm, a form of the proportional swap system, forces the path flow sequence to converge to a feasible state even without the monotonicity assumption (Mounce and Carey, 2011). The iterative sequence can be viewed as an evolutionary process of flow pattern from one disequilibrium state to an equilibrium or local stability state. To measure the convergence of this algorithm, a relative gap function is defined as Eq. (17). The pseudo-code of the TBCG algorithm is presented below in Algorithm 2.

$$
\operatorname{Regap}\left(k, f_{n}\right)=\max \left\{\frac{c_{p}^{r s}\left(k, f_{n}\right)-c_{\min }^{r s}\left(f_{n}\right)}{c_{\min }^{r s}\left(f_{n}\right)}\right\} \forall p \in\left\{i\left|f_{i}^{r s}(k)\right| 0, r s \in R S\right\}
$$

The proposed four strategies in the TBCG algorithm target at spatial and temporal exploration and exploitation to generate the most relevant path sets and time ranges that are fed into the route-swapping algorithm. The intrinsic feature of the route-swapping algorithm is shifting flows from time-dependent paths with high disutilities to low disutility paths. A BR-DUE state is achieved through iterative path generation and path-flow swapping processes provided that the flow adjustment parameters meet the convergence conditions (Nagurney and Zhang, 1997). The parameter setting and initial solution are two essential elements affecting the convergent flow patterns. According to Appendix A, it is likely that different inputs result in non-unique BR-DUE solutions. To measure the distance between two different BR-DUE solutions $f_{1}^{*}$ and $f_{2}^{*}$, the Euclidean distance can be adopted as $d\left(f_{1}^{*}, f_{2}^{*}\right)=\left\|f_{1}^{*}-f_{2}^{*}\right\|_{\ell^{2}}$, where $\|\cdot\|_{\ell^{2}}$ is a $\ell^{2}$-norm operator. To measure how good a BR-DUE solution is, the system cost, defined as the inner product of path flow and cost vectors, gives a yardstick. These two measures of distance have large implications for traffic prediction and control.

\subsection{A case for illustrating tolerance-based strategies}

We illustrate these strategies in a two-node network of three parallel links connecting one OD pair (Fig. 3). The link free flow times and capacities are given near the links. The link travel times associated with integer arrival time $k$ are calculated by the following iterative function (Liu et al., 2015)

$$
t_{a}(k, f)=\max \left\{t_{a}(k-1, f)+\eta_{1}\left(\frac{u_{a}(k)}{c_{a}}\right)^{\eta_{2}}, t_{a}^{0}\right\}
$$

where $u_{a}(k)$ is the flow of link $a$ during $k$; $t_{a}^{0}$ and $c_{a}$ are the free flow travel time and capacity of link $a ; \eta_{1}$ and $\eta_{2}$ are two parameters equal to 0.2 and 1 respectively. The targeted time interval range is from 1 min to $10 \mathrm{~min}$. 


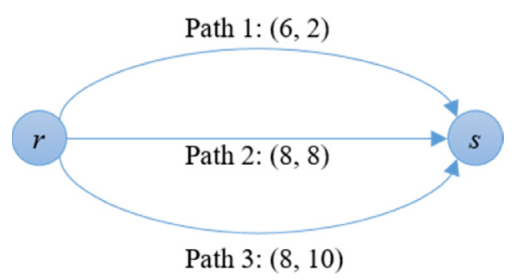

Fig. 3. A two-node network of three parallel links.

Table 2

The path flows and disutilities at each iteration.

\begin{tabular}{lllllllll}
\hline \multirow{2}{*}{ Iteration $\varepsilon_{n}^{\text {rs }}$} & Item & Path & \multicolumn{2}{l}{ Time intervals } \\
\cline { 5 - 9 } & & & 1 & 2 & 3 & 4 & 5 \\
\hline 0 & 0.2 & Disutility & 1 & 1.20 & 1.20 & 1.20 & 1.80 & 2.40 \\
1 & 0.2 & Path flow & 1 & 90.94 & 9.06 & 0 & - & - \\
& & Disutility & 2 & 4.35 & 5.20 & 5.60 & 6.00 & 6.40 \\
2 & 0.2 & Path flow & 1 & 42.38 & 0 & 0 & - & - \\
& & 2 & 28.81 & 28.81 & - & - & - \\
& & Disutility & 1 & 1.97 & 2.32 & 2.72 & 3.12 & 3.52 \\
& & & 2 & 1.66 & 1.92 & 2.32 & 2.80 & 3.40
\end{tabular}

\begin{tabular}{|c|c|c|c|c|c|c|c|c|c|c|c|c|c|}
\hline \multirow[t]{2}{*}{ Iteration $\varepsilon_{n}^{r s}$} & & \multirow[t]{2}{*}{ Item } & \multirow[t]{2}{*}{ Path } & \multicolumn{10}{|c|}{ Time intervals } \\
\hline & & & & 1 & 2 & 3 & 4 & 5 & 6 & 7 & 8 & 9 & 10 \\
\hline \multirow[t]{4}{*}{3} & 0.2 & Path flow & 1 & 23.21 & 17.31 & 0 & 0 & 0 & 0 & - & - & - & - \\
\hline & & & 2 & 16.43 & 16.43 & 16.43 & 10.21 & - & - & - & - & - & - \\
\hline & & Disutility & 1 & 1.62 & 1.93 & 2.03 & 2.23 & 2.43 & 2.63 & 2.83 & 3.03 & 3.23 & 3.43 \\
\hline & & & 2 & 1.64 & 1.68 & 1.73 & 1.94 & 2.14 & 2.34 & 2.54 & 2.80 & 3.10 & 3.40 \\
\hline \multirow[t]{4}{*}{4} & 0.1 & Path flow & 1 & 27.90 & 7.58 & 1.16 & 0 & 0 & 0 & - & - & - & - \\
\hline & & & 2 & 21.12 & 21.12 & 21.12 & 0 & - & - & - & - & - & - \\
\hline & & Disutility & 1 & 1.72 & 1.83 & 1.83 & 2.03 & 2.23 & 2.43 & 2.63 & 2.83 & 3.03 & 3.23 \\
\hline & & & 2 & 1.67 & 1.73 & 1.80 & 1.99 & 2.19 & 2.39 & 2.59 & 2.80 & 3.10 & 3.40 \\
\hline
\end{tabular}

(-: not exist in the PTPS).

Other parameters are set as: $Q^{r s}=100, \omega=0.5, \mu=0.5, \Delta_{1}=1 \mathrm{~min}, \alpha=0.2 \$ / \mathrm{min}, \beta=0.1 \$ / \mathrm{min}, \gamma=0.3 \$ / \mathrm{min}, \mathrm{k}^{r s *}=$ $10 \mathrm{~min}, \delta^{r s}=2 \min \varepsilon^{r s}=\varepsilon^{r s}=0.1, \varepsilon_{\max }^{r s}=0.2$. For initialization, we set $\Delta_{1}=2 \min , \varepsilon_{1}^{r s}=2, \Phi_{1}^{r s}=\{(1,1),(1,2),(1,3)\} P_{1}^{r s}=$ $\{1\}$ and $c^{r s}(k, 0)(k=1,2, \ldots, 5)$ as presented in Table 2 . Besides the equilibrium results at each iteration after running the TBCG algorithm, the table exhibits the changes of the relative convergence threshold and temporal resolution.

At the first iteration, no time-dependent path satisfies Eq. (12) after reassigning path flows on $\Phi_{1}^{r s}$, and the TBMDPSs are performed at all time intervals due to the large minimum disutility (4.35). As a result, path 2 is found and added to $P_{1}^{r s}$. To illustrate the process of each strategy more intuitively, the second iteration is presented using a timeline with time intervals shown above and path IDs on the left-side hand (Fig. 4). The travel disutilities and flows at each interval are listed below the timeline. Moreover, the current PTPS is displayed by the red segments; the new time-dependent paths satisfying Eq. (12) are displayed with yellow segments; and time intervals satisfying Eq. (16) are marked with rightward arrows. To explain strategy (iv), $c^{r s}(k, 0)$ is repeated after each time interval in Fig. 4(b).

At the second iteration, the time-dependent paths $(2,1)$ and $(2,2)$ have the minimum disutility (1.60) and fall out of $\Phi_{2}^{r s}$ as shown in Fig. 4(a). Consequently, both paths are added into $\Phi_{2}^{r s}$ during the temporal exploration. Then, the traffic assignment is implemented with the updated $\Phi_{2}^{r s}$ and the path disutilities are depicted in Fig. 4(b). Since the relative difference between the lower bound at interval $5(2.40)$ and the minimum disutility (1.66) is larger than $\varepsilon_{2}^{r s}$, time interval 5 is skipped when performing the TBMDPS. Afterwards, the temporal exploitation is triggered by decreasing $\Delta_{2}$ since no new path is generated. Fig. 4(c) presents the path disutilities, flows and PTPS under the new temporal resolution.

The traffic assignment and path search are conducted alternately at the last two iterations, in which $\varepsilon_{3}^{r s}$ is decreased at iteration 3 and the required equilibrium state is reached at the fourth iteration. It should be noted that path 3 has the same minimum disutility as path 2 (1.60 shown in Fig. 4(a)) since both paths have the same free flow time. As shown in Table 2 , the minimum disutility at the last iteration is 1.67. Path 3 cannot be identified through the path search, which demonstrates the difference between the classic shortest path algorithm and the TBMDPS.

\section{Numerical examples}

This section presents numerical examples to assess the proposed TBCG algorithm for BR-DUE. Three aspects are concerned: the influence of the TBMDPS, the results of TBCG algorithm for DUE and BR-DUE, and the improvements due to the strategies. The solution algorithm is run on a personal computer with an Intel ${ }^{(\mathrm{R})} \mathrm{Core}^{(\mathrm{TM})} \mathrm{i7}-67003.40 \mathrm{GHz} \mathrm{CPU}$ and 


\begin{tabular}{|c|c|c|c|c|c|c|c|}
\hline & the PTPS & & ) satis & . $(9)$ & $k \mathrm{sa}$ & Eq. (1 & \\
\hline \multirow{5}{*}{ (a) } & Interval & 1 & 2 & 3 & 4 & 5 & \\
\hline & [ Disutility & 4.35 & 5.20 & 5.60 & 6.00 & 6.40 & \\
\hline & [ Flow & 90.94 & 9.06 & 0 & 0 & 0 & ] \\
\hline & $\begin{array}{l}\text { Path } 2 \\
\text { [ Disutility }\end{array}$ & 1.60 & 1.60 & 2.20 & 2.80 & 3.40 & \\
\hline & [ Flow & 0 & 0 & 0 & 0 & 0 & ] \\
\hline
\end{tabular}

Temporal exploration

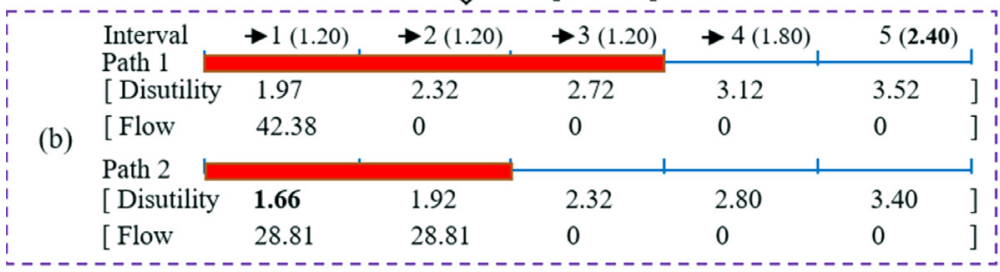

Temporal exploitation

\begin{tabular}{|c|c|c|c|c|c|c|c|c|c|c|c|}
\hline & Interval & 1 & 2 & 3 & 4 & 5 & 6 & 7 & 8 & 9 & 10 \\
\hline & [ Disutilit & y 1.58 & 1.97 & 2.12 & 2.32 & 2.52 & 2.72 & 2.92 & 3.12 & 3.32 & 3.52 \\
\hline \multirow[t]{3}{*}{ (c) } & [ Flow & 21.19 & 21.19 & 0 & 0 & 0 & 0 & 0 & 0 & 0 & 0 \\
\hline & $\begin{array}{l}\text { Path } 2 \\
\text { [ Disutilit }\end{array}$ & y 1. & 1.66 & 1.70 & 1.92 & 2.12 & 2.32 & 2.52 & 2.80 & 3.10 & 3.40 \\
\hline & [Flow & 14.41 & 14.41 & 14.41 & 14.41 & 0 & 0 & 0 & 0 & 0 & 0 \\
\hline
\end{tabular}

Fig. 4. Three strategies at the second iteration.

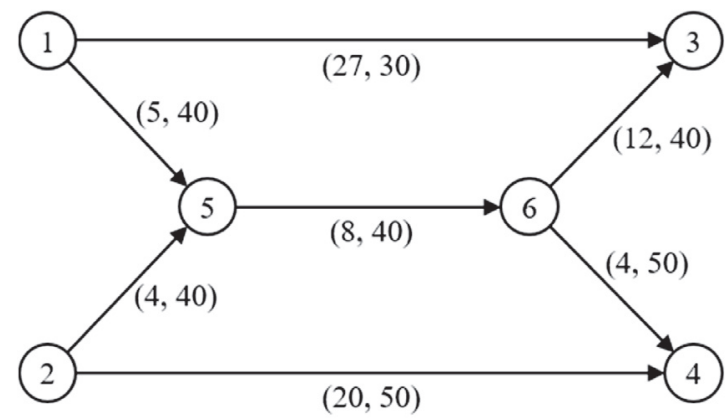

A path defined by a sequence of nodes

Path 1: 1-5-6-3

Path 2: 1-3

Path 3: 2-5-6-4

Path 4: 2-4

Fig. 5. The test network.

8.00 GB RAM. We set the parameters as $\omega=0.5$ and $\mu=0.5 ; \Delta=1 \mathrm{~min}$; penalty coefficients $\alpha=6.4 \$ / \mathrm{h}, \beta=3.9 \$ / \mathrm{h}$ and $\gamma=15.21 \$ / \mathrm{h} ; k^{r s *}=9 \mathrm{am}$ and $\delta^{r s}=0.1 \mathrm{~h}$; the interested time period is from 8 am to $12 \mathrm{pm} ; \varepsilon^{r s}=\varepsilon^{r s}=0.1$ and $\varepsilon_{1}^{r s}=\varepsilon_{\text {max }}^{r s}=$ 0.2 unless otherwise explained. The link travel times are calculated by Eq. (18).

\subsection{Example 1: six-node network}

The six-node test network (Shao et al., 2013) is adopted for illustrating the effects of strategy ( $i$ ) on path generation and the different solutions to the TBCG algorithm for DUE and BR-DUE. As shown in Fig. 5, this network has two OD pairs, six nodes, seven links, and four paths.

To illustrate the difference derived from the TBMDPS strategy, we set the demand for OD pairs $(1,3),(2,4)$ as 1600 and 0 units respectively. Fig. 6 indicates that the TBCG algorithm results in different equilibrium solutions compared with the traditional CG algorithm. As shown in Fig. 6(a) and (b), only path 1 is found and used for BR-DUE assignment. Recall that only the paths satisfying Eq. (11) are added to the path set. Path 2 does not satisfy this condition, although its disutility is smaller than path 1 in several intervals. This conclusion is deduced from Fig. 6(c) and (d), where two paths are generated and have non-zero path flows when $\varepsilon^{r s}$ is equal to zero.

When the demand for OD pairs $(1,3)$ and $(2,4)$ increase to large values, such as $Q^{13}=10^{4}$ and $Q^{24}=8000$, traffic congestion occurs in this network. Some travelers depart early and shift paths to avoid late arrival penalty. Taking OD pair (1, 


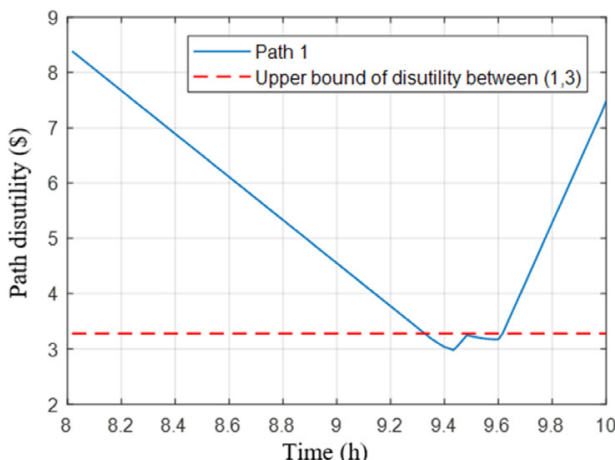

(a) Path disutility when $\epsilon^{r s}=0.1$

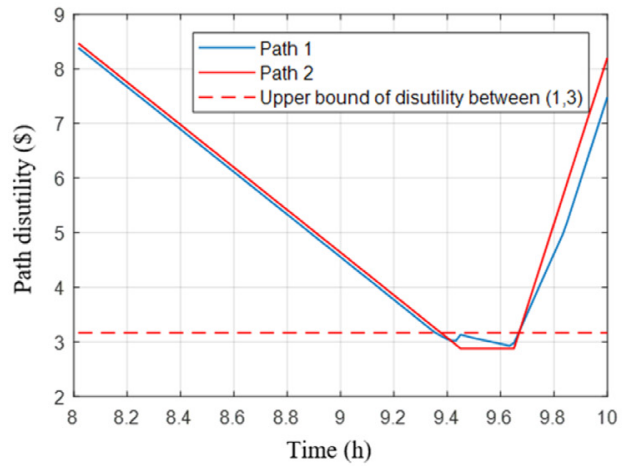

(c) Path disutility when $\epsilon^{r s}=0$

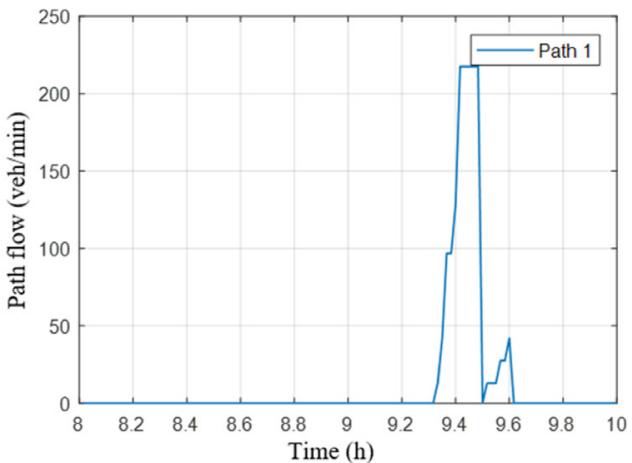

(b) Path flow when $\epsilon^{r s}=0.1$

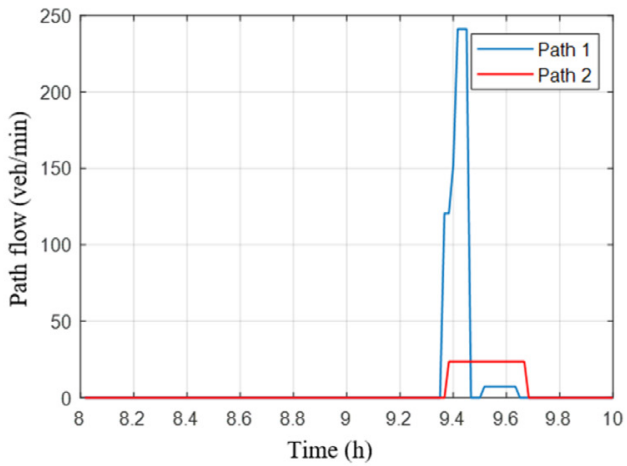

(d) Path flow when $\epsilon^{r s}=0$

Fig. 6. Equilibrium solution for BR-DUE.

3) for example, in Fig. 6(b), travelers are assigned to the time period [9.3 am, $9.6 \mathrm{am}$ ] on path 1 when the OD demand is small, while this period shifts to [8.7 am, $9.3 \mathrm{am}$ ] on path 1 and path 2 as depicted in Fig. 7(d). Moreover, the equilibrium solution of the TBCG algorithm for BR-DUE model is different from that of the DUE. At the steady state of DUE, disutilities of used time-dependent paths are equal for the same OD pair and the unused time-dependent paths have larger disutilities. These results are consistent with the DUE condition as depicted in Fig. 7(a) and (b). Regarding the BR-DUE, disutilities of the used time-dependent paths for the same OD pair are different and no larger than the critical values defined by Eq. (12), which are shown with the red dashed line in Fig. 7(c). Fig. 7 indicates that the TBCG algorithm is capable of solving the BR-DUE problem.

\subsection{Example 2: the Nguyen and Dupuis network}

This example assesses the impact of the strategies (ii) - $(v)$ on the improvement of the CG algorithm under different tolerances. For a fair comparison, the CG algorithms with and without these strategies both use the TBMDPS to generate paths. As depicted in Fig. 8, this network (Long et al., 2013b; Li et al., 2018) has 13 nodes and four OD pairs including $(1,2)$, $(1,3),(4,2)$ and $(4,3)$ with different traffic demand of 15,360, 5120, 5120 and 8960 respectively. The link free flow times and capacities are marked near the corresponding links. The temporal resolution is initialized to $\Delta_{1}=2$ and $\omega=0.1$ in this example. For the route-swapping process, the step-size rule $\rho \cdot(1+\eta \sigma) / 1+\tau / 2000$ is adopted, where $\rho=0.1$ ensures a steady convergence, $\sigma$ denotes the number of temporal exploitations, $\eta$ is a re-scaling factor, and $\tau$ is the number of path flow reassignments at each outer iteration.

The TBCG algorithm generates paths for traffic assignment driven by the tolerance-based travel behavior. The number of generated paths at BR-DUE is far less than that by path enumeration, which significantly decreases the size of timedependent paths. Fig. 9 presents the comparison of the number of paths derived from the TBCG algorithm and path enumeration. It shows the effectiveness even when traffic demand is large in this example.

Table 3 shows the numbers of path searches, network loadings, and computation times of the CG algorithms with and without strategies (ii) - (iv) under a series of tolerances $\varepsilon^{r s}$. Compared with the CG algorithm without these strategies, strategy (ii) takes fewer network loadings and computation time to obtain the equilibrium results. More than 20 seconds are saved, although the differences of computation times decrease with the increase of $\varepsilon^{\text {rs }}$. Strategy (iii) increases the number of network loadings for some $\varepsilon^{r s}$. However, it gains more than 1.5 times speedup in computation time due to reduced time- 


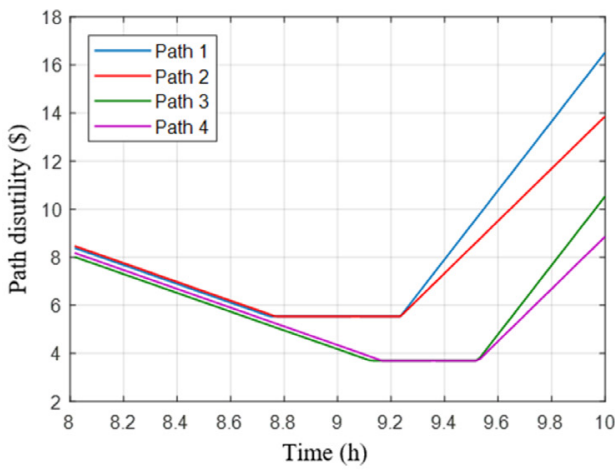

(a) Path disutility for DUE

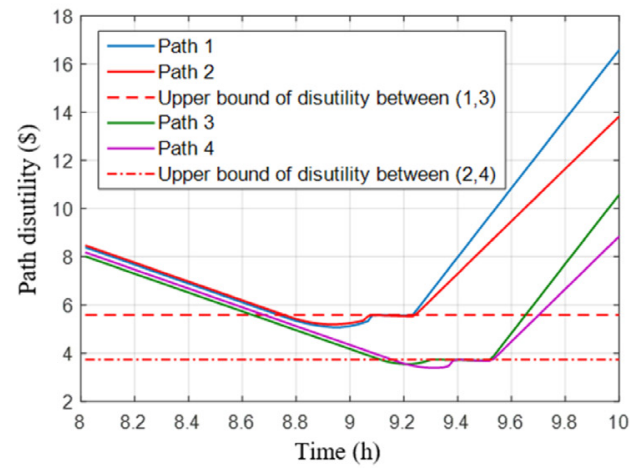

(c) Path disutility for BR-DUE

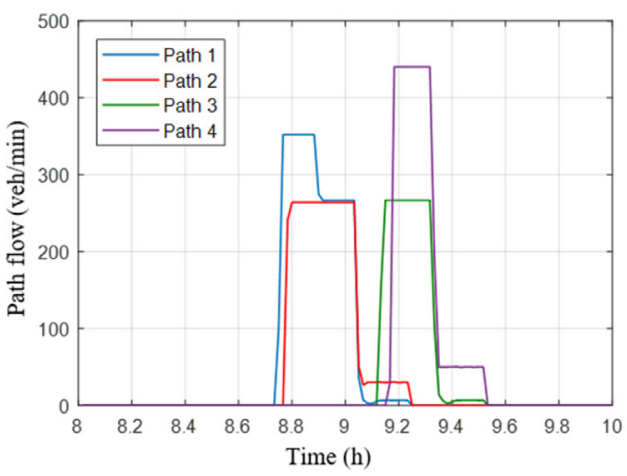

(b) Path flow for DUE

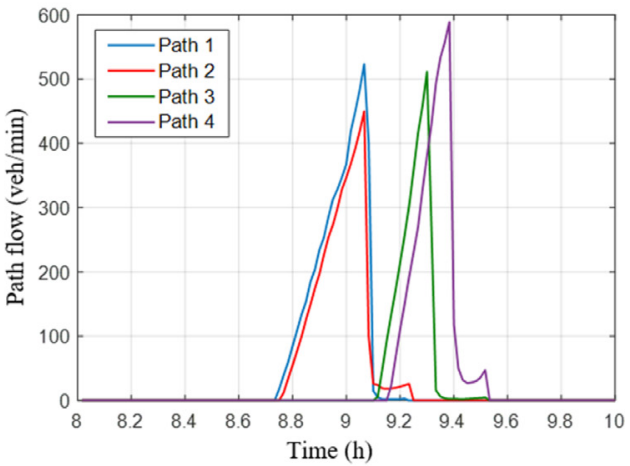

(d) Path flow for BR-DUE

Fig. 7. Equilibrium solutions for BR-DUE and DUE.

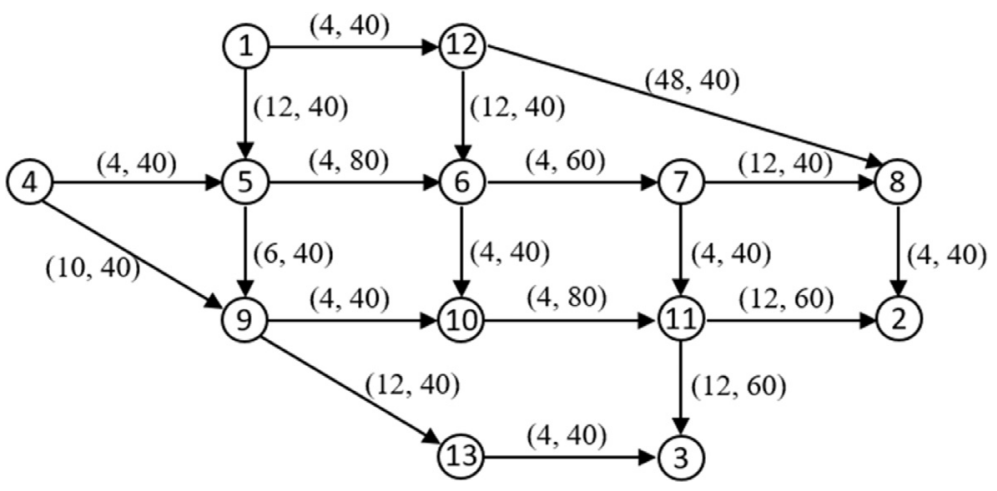

Fig. 8. The Nguyen and Dupuis network.

dependent paths when $\Delta_{n}=2 \mathrm{~min}$. Strategy (iv) skips around 150 tolerance-based path searches. Incorporating all strategies, the TBCG algorithm improves the CG algorithm in all aspects, leading to speedup factors larger than 2.

To demonstrate the effects of the spatial and temporal parameters of the four strategies, we fix other parameters and show the results in Table 4. As shown in the first block, a larger $\Delta_{1}$ leads to less dynamic network loadings and path searches, and hence a larger speedup factor. $\omega$ impacts the occurrences of leaps from $\Delta_{1}$ to $\Delta$. Although more leaps are needed when $\omega$ increases, the number of dynamic network loadings and computation time decrease. The reason is that decreasing $\Delta_{n}$ may generate new paths, which needs more path flow assignments to reach a new equilibrium state. $\Delta$ is related to the convergence precision. Decreasing $\Delta$ results in dramatically increases of the computation time of the CG algorithm, compared with the TBCG algorithm. $\varepsilon_{\max }^{\text {rs }}$ and $\varepsilon^{\text {rs }}$ are related to the path flow assignment and path search process respectively. Larger $\varepsilon_{\max }^{r s}$ ensures the route-swapping algorithm to achieve the stop criterion with less iterations. However, this comes at the expense of current precision, as it may need extra iterations to generate new paths and perform path flow reassignments (e.g. $\varepsilon_{\max }^{r s}=0.25$ ). When $\varepsilon^{r s}$ taking a small value, more paths may satisfy Eq. (11) and be added to the 


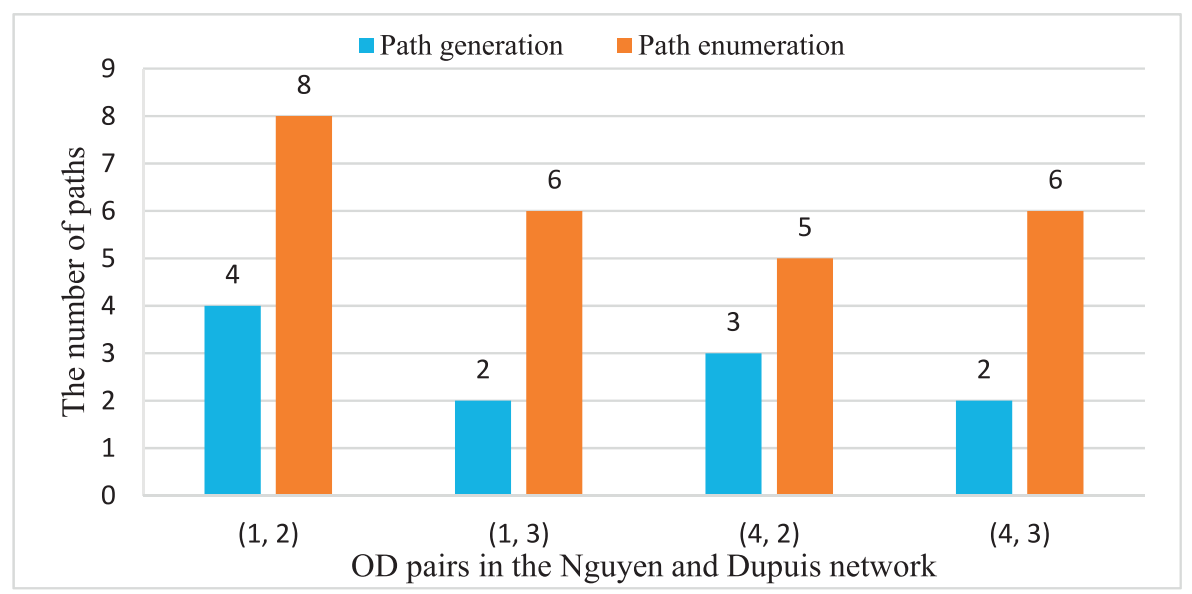

Fig. 9. Comparison of the number of paths.

Table 3

Improvements of different strategies on CG algorithm with a range of tolerances $\varepsilon^{r s}$.

\begin{tabular}{|c|c|c|c|c|c|c|c|}
\hline \multirow[t]{2}{*}{ Strategies } & \multirow[t]{2}{*}{ Items } & \multicolumn{6}{|l|}{$\varepsilon^{r s}$} \\
\hline & & 0.05 & 0.06 & 0.07 & 0.08 & 0.09 & 0.10 \\
\hline \multirow[t]{3}{*}{ CG algorithm without strategy (ii)-(iv) } & Number of path searches & 722 & 722 & 722 & 722 & 722 & 722 \\
\hline & Number of network loadings & 1855 & 1338 & 1217 & 1116 & 1103 & 931 \\
\hline & Computation time $(\mathrm{s})$ & 298.57 & 234.41 & 190.30 & 174.61 & 167.19 & 139.28 \\
\hline \multirow[t]{2}{*}{ Strategy (ii) } & Number of network loadings & 1125 & 1000 & 866 & 907 & 737 & 687 \\
\hline & Computation time (s) & 191.11 & 161.75 & 139.91 & 141.67 & 120.75 & 118.62 \\
\hline \multirow[t]{3}{*}{ Strategy (iii) } & Number of network loadings & 2270 & 1847 & 1531 & 1082 & 986 & 854 \\
\hline & Computation time $(\mathrm{s})$ & 182.34 & 150.28 & 118.73 & 90.03 & 79.82 & 69.20 \\
\hline & Speedup factor & 1.64 & 1.56 & 1.60 & 1.94 & 2.09 & 2.01 \\
\hline Strategy (iv) & Number of path searches & 567 & 573 & 575 & 576 & 573 & 578 \\
\hline \multirow[t]{4}{*}{ TBCG algorithm } & Number of path searches & 672 & 674 & 669 & 668 & 670 & 673 \\
\hline & Number of network loadings & 861 & 707 & 687 & 686 & 651 & 608 \\
\hline & Computation time (s) & 108.98 & 82.34 & 77.53 & 76.56 & 72.02 & 63.80 \\
\hline & Speedup factor & 2.74 & 2.85 & 2.45 & 2.28 & 2.32 & 2.18 \\
\hline
\end{tabular}

path set for traffic assignment. As demonstrated, the TBCG algorithm obtains speedup factors significantly larger than 2 with most of the parameter setups.

\subsection{Example 3: larger networks}

The properties of path disutility function (Eq. (2)) make the projection-based algorithms used in, for example, Chen et al. (2001) and Long et al. (2013b), ineffective for DTA in large networks. This subsection uses larger networks, i.e. Sioux Falls network, Eastern Massachusetts (EMA) network, Anaheim network, and Chicago-Sketch network to illustrate the effectiveness of the TBCG algorithm in terms of computation time and the number of dynamic network loadings. The Sioux Falls network consists of 24 nodes and 76 links, the EMA network has 74 nodes and 258 links, the Anaheim network contains 416 nodes and 914 links, and the Chicago-Sketch network has 933 nodes and 2950 links. The network topology and OD demands are obtained from http://www.bgu.ac.il/ bargera/tntp/. We perform some data transformation on the free-flow travel times and link capacities to fit the randomly selected OD pairs. Compared with the settings of Example 2, the same stepsizes are adopted in the route-swapping process except for $\rho$. The small demand result in a larger $\rho(0.5)$ in this example to ensure an acceptable convergence. Other settings remain the same as Example 2.

As an illustration of the convergence process, Fig. 10 shows the convergence curves of the original CG algorithm and the TBCG algorithm in the Anaheim network with 1406 OD pairs. As depicted, the CG algorithm generates new paths only when the relative gap is less than 0.1, and it takes 38 path flow reassignments. For the TBCG algorithm, new path generation and temporal exploitation occur after 16 and 30 path flow reassignments respectively. Note that both processes are performed in a low temporal resolution. Moreover, the TBCG algorithm needs less path flow reassignments to converge to BR-DUE. A low temporal resolution guarantees faster convergence per iteration and a larger convergence threshold ensures less path flow reassignments, which lead to 2.14 speedup factor compared to the original CG algorithm.

Table 5 provides the number of dynamic network loadings and the computation times required for both the original CG algorithm and the TBCG algorithm to achieve the BR-DUE. For different network configurations, the demands are enlarged from the original demands to produce congestion effects. It shows that the TBCG algorithm outperforms the original CG 
Table 4

Effects of the parameters of the TBCG algorithm.

\begin{tabular}{|c|c|c|c|c|c|c|c|c|c|}
\hline \multicolumn{5}{|c|}{ Parameters for comparison } & \multirow[t]{2}{*}{$\begin{array}{l}\text { CG algorithm } \\
\text { Computation } \\
\text { time }(\mathrm{s})\end{array}$} & \multicolumn{3}{|c|}{ TBCG algorithm } & \multirow[t]{2}{*}{$\begin{array}{l}\text { Speedup } \\
\text { factor }\end{array}$} \\
\hline$\Delta_{1}$ & $\omega$ & $\Delta$ & $\varepsilon_{\max }^{r s}$ & $\varepsilon^{r s}$ & & $\begin{array}{l}\text { Number of } \\
\text { network } \\
\text { loadings }\end{array}$ & $\begin{array}{l}\text { Number of path } \\
\text { searches }\end{array}$ & $\begin{array}{l}\text { Computation } \\
\text { time }(\mathrm{s})\end{array}$ & \\
\hline 1 & 0.5 & 0.5 & 0.2 & 0.1 & & 697 & 1336 & 162.60 & 2.95 \\
\hline 2 & 0.5 & 0.5 & 0.2 & 0.1 & 479.85 & 614 & 1215 & 96.37 & 4.98 \\
\hline 4 & 0.5 & 0.5 & 0.2 & 0.1 & & 584 & 1137 & 48.69 & 9.85 \\
\hline 4 & 0.125 & 0.5 & 0.2 & 0.1 & & 656 & 851 & 96.80 & 4.96 \\
\hline 4 & 0.25 & 0.5 & 0.2 & 0.1 & 479.85 & 638 & 1042 & 79.62 & 6.03 \\
\hline 4 & 0.5 & 0.5 & 0.2 & 0.1 & & 584 & 1137 & 47.46 & 10.11 \\
\hline 4 & 0.5 & 0.5 & 0.2 & 0.1 & 479.85 & 584 & 1137 & 47.46 & 10.11 \\
\hline 4 & 0.5 & 1 & 0.2 & 0.1 & 182.05 & 614 & 594 & 40.01 & 4.55 \\
\hline 4 & 0.5 & 2 & 0.2 & 0.1 & 98.16 & 644 & 325 & 36.70 & 2.67 \\
\hline 2 & 0.5 & 1 & 0.15 & 0.1 & & 870 & 653 & 83.76 & 1.97 \\
\hline 2 & 0.5 & 1 & 0.2 & 0.1 & & 608 & 673 & 63.80 & 2.58 \\
\hline 2 & 0.5 & 1 & 0.25 & 0.1 & 164.68 & 754 & 674 & 99.71 & 1.65 \\
\hline 2 & 0.5 & 1 & 0.3 & 0.1 & & 507 & 695 & 59.49 & 2.77 \\
\hline 2 & 0.5 & 1 & 0.35 & 0.1 & & 424 & 707 & 52.92 & 3.11 \\
\hline 2 & 0.5 & 1 & 0.4 & 0.1 & & 374 & 709 & 47.05 & 3.50 \\
\hline 2 & 0.5 & 1 & 0.2 & 0.05 & & 651 & 650 & 72.53 & 2.49 \\
\hline 2 & 0.5 & 1 & 0.2 & 0.1 & & 608 & 673 & 63.80 & 2.83 \\
\hline 2 & 0.5 & 1 & 0.2 & 0.15 & 180.51 & 608 & 673 & 64.30 & 2.81 \\
\hline 2 & 0.5 & 1 & 0.2 & 0.2 & & 635 & 677 & 64.87 & 2.78 \\
\hline 2 & 0.5 & 1 & 0.2 & 0.25 & & 508 & 614 & 50.76 & 3.56 \\
\hline 2 & 0.5 & 1 & 0.2 & 0.3 & & 599 & 712 & 57.90 & 3.12 \\
\hline
\end{tabular}

(Fixed parameter: $\mu=0.1, \varepsilon^{r s}=0.1$. In each block, the parameter values in bold are the focus of attention).

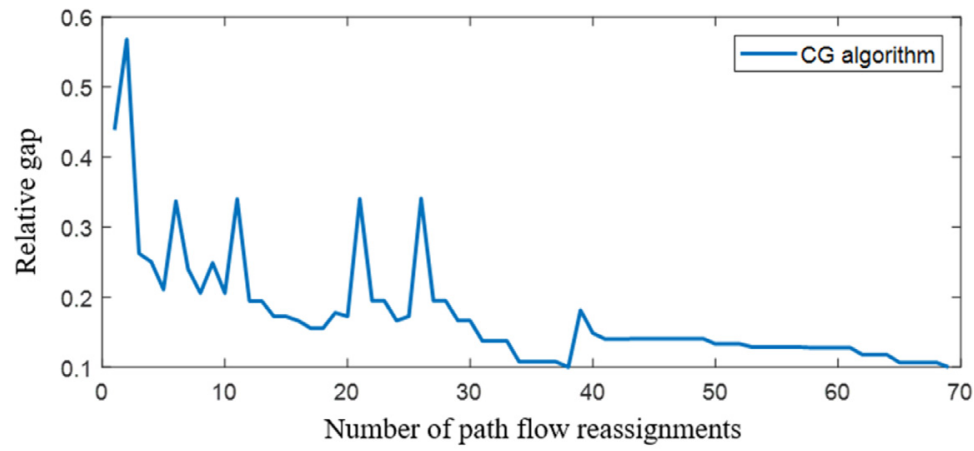

(a) Convergence of the $\mathrm{CG}$ algorithm

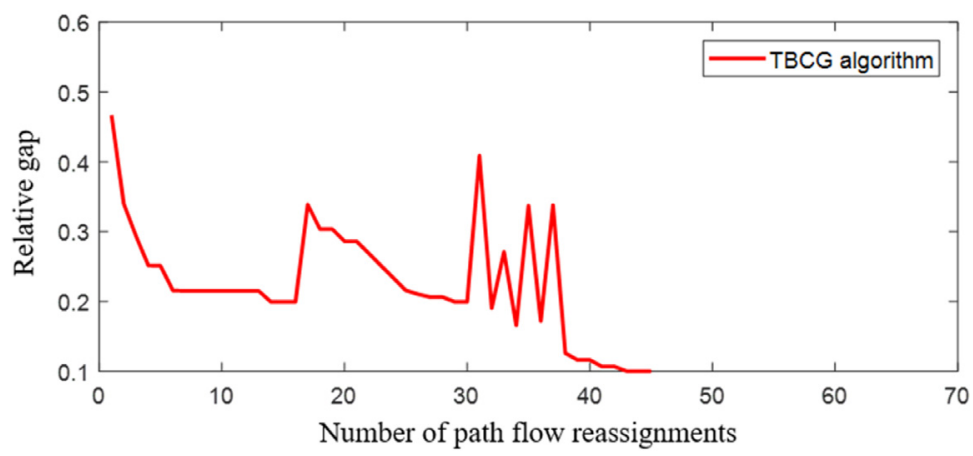

(b) Convergence of the TBCG algorithm

Fig. 10. Convergence of two algorithms. 
Table 5

Performance of the TBCG algorithm.

\begin{tabular}{|c|c|c|c|c|c|c|c|c|}
\hline \multirow[t]{2}{*}{ Network } & \multirow[t]{2}{*}{$\begin{array}{l}\text { Demand } \\
\text { (*original } \\
\text { demand) }\end{array}$} & \multirow[t]{2}{*}{$\begin{array}{l}\text { OD } \\
\text { pairs }\end{array}$} & \multicolumn{3}{|c|}{ Number of network loadings } & \multicolumn{3}{|c|}{ Computation time (s) } \\
\hline & & & $\begin{array}{l}\text { CG } \\
\text { algorithm }\end{array}$ & $\begin{array}{l}\text { TBCG } \\
\text { algorithm }\end{array}$ & $\begin{array}{l}\text { Reductions } \\
(\%)\end{array}$ & $\begin{array}{l}\text { CG } \\
\text { algorithm }\end{array}$ & $\begin{array}{l}\text { TBCG } \\
\text { algorithm }\end{array}$ & $\begin{array}{l}\text { Speedup } \\
\text { factor }\end{array}$ \\
\hline \multirow[t]{4}{*}{ Sioux Falls } & 1 & 50 & 870 & 777 & 10.69 & 279.73 & 146.14 & 1.91 \\
\hline & & 100 & 1232 & 875 & 28.98 & 1165.24 & 476.72 & 2.44 \\
\hline & 2 & 50 & 1294 & 827 & 36.09 & 520.4 & 262.76 & 1.98 \\
\hline & & 100 & 1620 & 1197 & 26.11 & 1565.71 & 730.63 & 2.14 \\
\hline \multirow[t]{4}{*}{ EMA } & 10 & 100 & 219 & 193 & 11.87 & 712.55 & 359.05 & 1.98 \\
\hline & & 200 & 545 & 509 & 6.61 & 2160.49 & 1060.17 & 2.04 \\
\hline & 20 & 100 & 309 & 231 & 25.24 & 546.01 & 272.86 & 2.00 \\
\hline & & 200 & 386 & 303 & 21.50 & 1799.14 & 823.95 & 2.18 \\
\hline \multirow[t]{5}{*}{ Anaheim } & 1 & 1406 & 74 & 57 & 22.97 & 3116.79 & 1459.8 & 2.14 \\
\hline & 10 & 200 & 149 & 78 & 47.65 & 807.25 & 304.84 & 2.65 \\
\hline & & 400 & 121 & 79 & 34.71 & 1247.66 & 612.33 & 2.04 \\
\hline & 20 & 200 & 323 & 193 & 40.25 & 2334.37 & 980.02 & 2.38 \\
\hline & & 400 & 318 & 172 & 45.91 & 6236.97 & 2769.73 & 2.25 \\
\hline \multirow{6}{*}{$\begin{array}{l}\text { Chicago- } \\
\text { Sketch }\end{array}$} & 20 & 600 & 112 & 77 & 31.25 & $13,940.34$ & 7004.20 & 1.99 \\
\hline & & 1000 & 216 & 142 & 34.26 & $28,363.46$ & $13,833.80$ & 2.05 \\
\hline & 50 & 200 & 112 & 72 & 35.71 & 3824.29 & 1810.8 & 2.11 \\
\hline & & 400 & 163 & 93 & 42.94 & 9432.38 & 4438.74 & 2.13 \\
\hline & 100 & 100 & 162 & 96 & 40.74 & 2941.28 & 1203.57 & 2.44 \\
\hline & & 200 & 188 & 90 & 52.13 & 5990.14 & 1937.03 & 3.09 \\
\hline
\end{tabular}
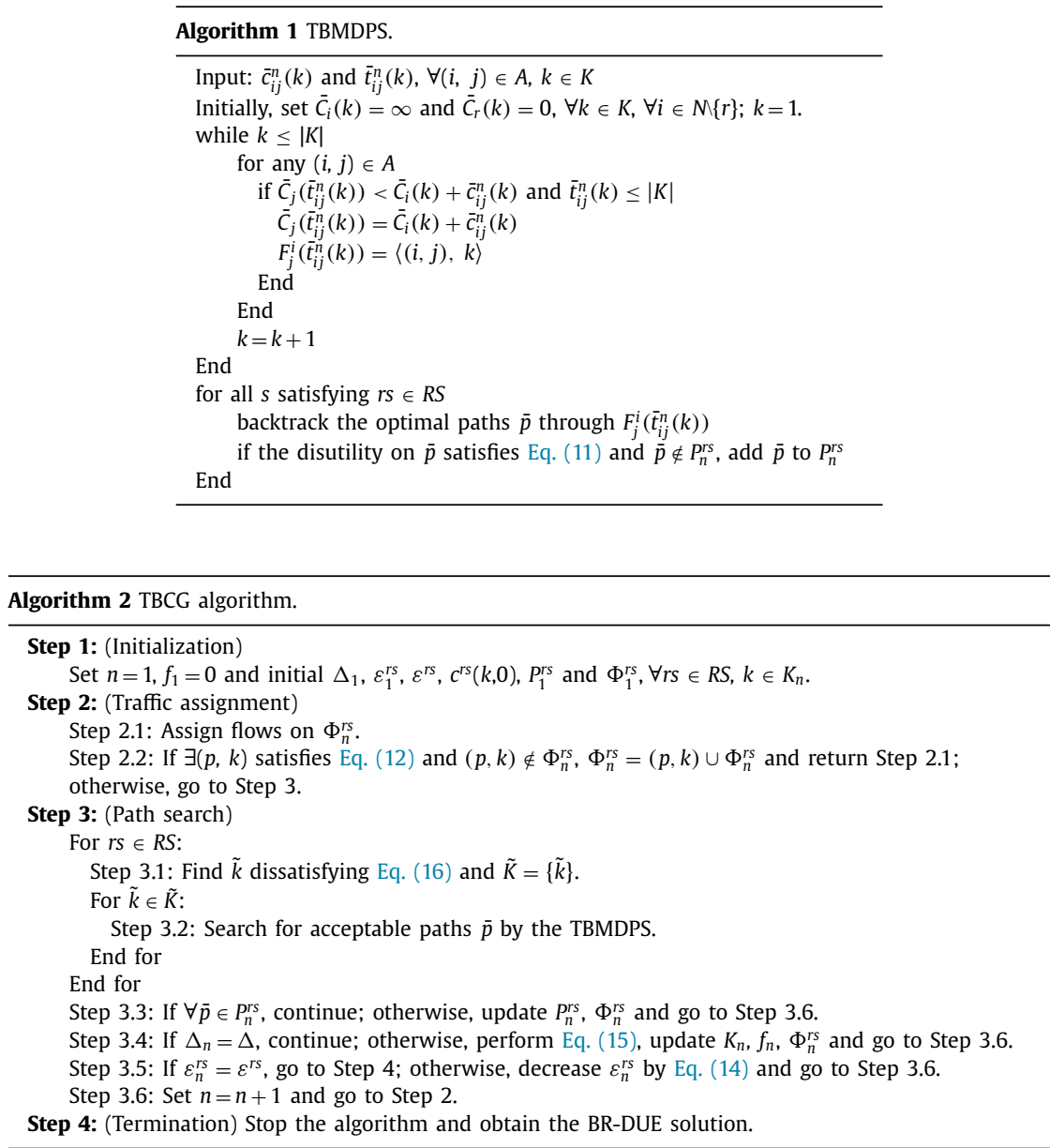


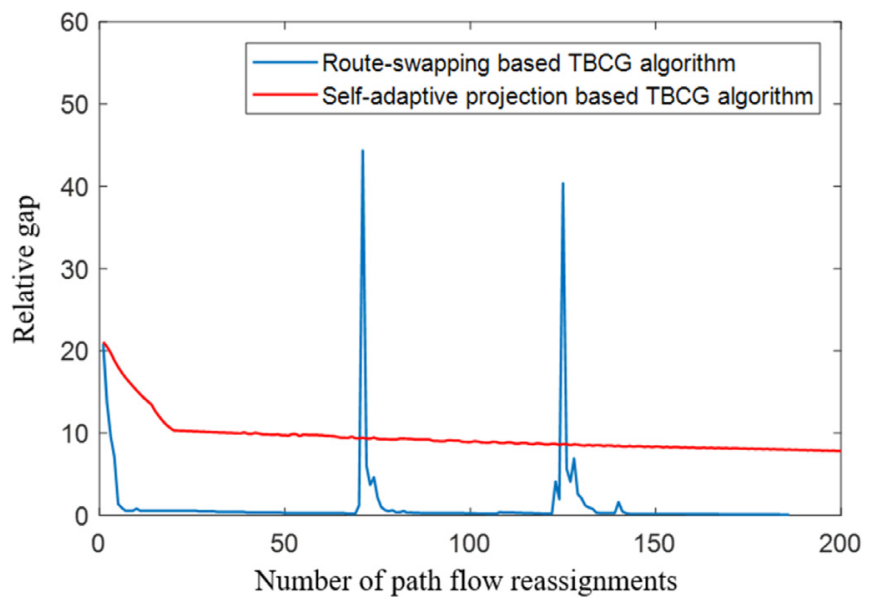

Fig. 11. Comparison between the route-swapping and self-adaptive projection algorithms.

algorithm in terms of both indicators. The speedup factors of computation time are ranged from 1.91 to 3.09 . In combination with Example 2, we can conclude that the speedup factors are significant and quite stable.

As an example to illustrate the flexibility of the TBCG algorithm, the self-adaptive projection method that is positioned in Han et al. (2015) with weak convergence condition and fast convergence is embedded into the TBCG scheme and compared with the route-swapping based TBCG algorithm. For a fair comparison, the same gap function shown in Eq. (17) is adopted in the EMA network with randomly selected 100 OD pairs. As depicted in Fig. 11, the route-swapping based TBCG algorithm converges to a stable state with around 180 path flow reassignments crossing two major fluctuations, which manifest path generation and temporal exploitation. Regarding the self-adaptive projection based TBCG algorithm, the relative gap is still large after 200 path flow reassignments although it decreases steadily. The total computation times are $413.17 \mathrm{~s}$ for the route-swapping based algorithm and $672.72 \mathrm{~s}$ for the self-adaptive projection based algorithm respectively. Thus, the routeswapping based TBCG algorithm performs better in this example.

\section{Conclusions and future research}

This paper proposed four tolerance-based strategies to extend the column generation (CG) algorithm for solving the bounded rational dynamic user equilibrium problem (BR-DUE). Incorporating the characteristics of BR, the strategies combine the spatial-temporal exploration and exploitation of flow patterns for finding the BR-DUE solutions. It is notable that the four strategies maintain the convergence property of the CG algorithm. In particular, under the monotonicity assumption of the path disutility, the proposed tolerance-based column generation (TBCG) algorithm is convergent. As illustrated in the numerical examples, the four strategies overall accelerate the original CG algorithm and reduce the numbers of path searches and dynamic network loadings. The TBCG algorithm is more efficient due to the smaller size of the path sets at most iterations.

On the basis of the TBCG algorithm, several extensions are worthy of exploring in future studies. First, travel demand in this study was assumed to be constants. However, this assumption is unlikely or even logically impossible in reality. Elastic demand or uncertain demand should be investigated. Second, the temporal resolution mainly increases in the TBCG algorithm for exploitation. Decreasing resolutions may also be considered in the temporal exploration process. Third, different travelers have different preferences. The trip purposes may lead to different values of time, early and late penalties. Hence, heterogeneity should be considered in the traffic assignment models as well. Lastly, as travel can be conceptualized as the derived demand from conducting activities at the destinations, the TBCG algorithm will be applied and further developed to address path generation and personalized network formation in multi-state supernetwork models (Liao et al., 2010, 2011, 2013; Liao, 2016) that are dedicated for activity-based travel demand analysis.

\section{Acknowledgments}

This work is jointly supported by the Fundamental Research Funds for the Central Universities (2018YJS095), the National Natural Science Foundation of China (71621001, 71771019), and the Netherlands Organization for Scientific Research (NWO). The first author is grateful to the financial support by the China Scholarship Council (CSC). 
Appendix A. Analyses of the existence and non-uniqueness of the solutions to the discrete time $\operatorname{VI}(f, \Omega)$ problem Eqs. (7)-(9)

Existence: According to Theorem 5.6 proposed by Han et al. (2015), the sufficiency for the existence of BR-DUE includes two conditions, which are both satisfied in this study.

First, $\tilde{c}_{p}^{r s}(k, f)$ is continuous with path flows.

This condition has been shown to be true for the VI problems in the continuous time domain, of which the dynamic network loading can be performed based on the Vickrey model (Han et al., 2013) and LWR-Lax model (Bressan and Han, 2013), etc. Regarding the finite-dimensional VI problem, the continuity of $\tilde{c}_{p}^{r s}(k, f)$ depends on the continuity of $c_{p}^{r s}(k, f)$, which has also been shown by Long et al. (2013a) through linear interpolation of link travel times.

Second, for each $r s \in R S$, let path travel disutility $c_{p}^{r s}(k, f)$ be decomposed into two additive components, i.e. $\psi_{r s}(\cdot)$ and $\varphi_{r s}(\cdot)$; moreover, the former one is monotonically increasing and the latter is Lipschitz continuous with constant $L_{r s}$.

This condition holds for the path disutility formulated in Eq. (2) with $\gamma>\alpha>\beta>0$ by supposing $\varphi_{r s}(k)=-\alpha k$ and

$$
\psi_{r s}\left(k+t_{p}^{r s}(k, f)\right)=\alpha\left(k+t_{p}^{r s}(k, f)\right)+ \begin{cases}\beta\left[k^{r s *}-\delta^{r s}-k-t_{p}^{r s}(k, f)\right] & \text { if } k+t_{p}^{r s}(k, f)<k^{r s *}-\delta^{r s} \\ \gamma\left[k+t_{p}^{r s}(k, f)-k^{r s *}-\delta^{r s}\right] & \text { if } k+t_{p}^{r s}(k, f)>k^{r s *}+\delta^{r s} \\ 0 & \text { otherwise }\end{cases}
$$

Non-uniqueness: As proved by Szeto and Lo (2006) and Han et al. (2015), the solutions to the BR-DUE problem are non-unique. Specifically, when the threshold of the acceptable relative difference $\varepsilon^{\text {rs }}$ approaches infinity, it is obvious that any path flow vector in $\Omega$ satisfies Eq. (6). On the contrary, the BR-DUE problem is degenerated to the general DUE when $\varepsilon^{r s}$ equals zero. The uniqueness of DUE solutions requires the path disutilities to be strictly monotone with path flow, which do not hold in general (Huang and Lam, 2002; Mounce and Carey, 2015).

Based on the above analyses, we confirm the existence and non-uniqueness of the solutions to the discrete time $\operatorname{VI}(f$, $\Omega$ ) problem.

\section{Appendix B. Proof of Theorem 1}

Proof. Instead of using Eq. (10) as the criterion to add new paths, strategy ( $i$ ) adopts Eq. (11) to model travelers' BR behavior for path choice. When $\varepsilon^{r s}$ equals zero, this strategy is degenerated to the general minimum disutility path search method. Let $P_{n}^{r s \prime}$ denote the path set, of which the members satisfy Eq. (10) but violate Eq. (11). Based on strategy ( $i$ ), these paths do not belong to $P_{n}^{r s}$ despite having lower path disutilities. The minimum disutility of paths in $P_{n}^{r s \prime}$, denoted by $c_{\text {min }}^{r s^{\prime}}\left(f_{n}\right)$, has a lower bound as follows

$$
c_{\min }^{r s^{\prime}}\left(f_{n}\right)>\left(1-\varepsilon^{r s}\right) \cdot c_{\min }^{r s}\left(f_{n}\right)
$$

where $c_{\min }^{r s}\left(f_{n}\right)$ is the minimum disutility of paths in $P_{n}^{r s}$.

The relative gap of the path disutility in path set $P_{n}^{r s \prime} \cup P_{n}^{r s}$ can be expressed as

$$
\begin{aligned}
\frac{c_{p}^{r s}\left(k, f_{n}\right)-c_{\min }^{r s^{\prime}}\left(f_{n}\right)}{c_{\min }^{r s^{\prime}}\left(f_{n}\right)} & <\frac{c_{p}^{r s}\left(k, f_{n}\right)-\left(1-\varepsilon^{r s}\right) \cdot c_{\min }^{r s}\left(f_{n}\right)}{\left(1-\varepsilon^{r s}\right) \cdot c_{\min }^{r s}\left(f_{n}\right)} \\
& =\frac{c_{p}^{r s}\left(k, f_{n}\right)-c_{\min }^{r s}\left(f_{n}\right)}{c_{\min }^{r s}\left(f_{n}\right)}+\frac{\varepsilon^{r s} \cdot c_{p}^{r s}\left(k, f_{n}\right)}{\left(1-\varepsilon^{r s}\right) \cdot c_{\min }^{r s}\left(f_{n}\right)} \\
& \leq \varepsilon_{n}^{r s}+\frac{\varepsilon^{r s} \cdot\left(1+\varepsilon_{n}^{r s}\right)}{1-\varepsilon^{r s}} \forall p \in P_{n}^{r s^{\prime}} \cup P_{n}^{r s}, k \in K_{n}
\end{aligned}
$$

The last inequality is derived from Eq. (12). $P_{n}^{r s \prime} \cup P_{n}^{r s}$ is the newly generated path set if we use the minimum disutility path search method instead of the TBMDPS and inequality (B.2) gives the corresponding relative convergence threshold. That is, strategy $(i)$ is equivalent to the minimum path search method combined with a $\varepsilon^{r s}$-related relative convergence threshold. Thus, strategy $(i)$ does not really relax the condition of convergence.

Regarding strategy (ii) and (iii), $\varepsilon_{n}^{r s}, \Delta_{n}$ and $K_{n}$ at the intermediate iterations are constants and have no influence on the convergence of the original CG algorithm. At the last iteration, $\varepsilon_{n}^{r s}$ and $\Delta_{n}$ are equal to the required values to obtain the BR-DUE solution. Analogous to the spatial path extension, temporal exploration is the extension in the temporal dimension that only occurs at the intermediate iterations. For time intervals satisfying Eq. (16), the free flow path disutility are larger than the upper bound of the tolerance disutility. Strategy (iv) skips these unnecessary path searches and thus has no effect on path generation. In sum, none of the strategies modifies the convergence conditions of the original CG algorithm.

\section{Appendix C. Proof of Corollary 1}

Proof. The proof is based on the conclusions in the studies of Leventhal et al. (1973) and Smith (1984), who have analyzed the convergence of the CG algorithm and the proportional swap system respectively. 
First, under the monotonicity assumption of the path disutility, the dynamical proportional swap system of $\mathrm{VI}\left(f_{n}, \Omega_{n}\right)$ is convergent to an equilibrium, where $\operatorname{VI}\left(f_{n}, \Omega_{n}\right)$ is the sub-problem of the $\operatorname{VI}(f, \Omega)$ Eqs. (7)-(9) at iteration $n$ and written as

$$
\begin{aligned}
& \sum_{r s \in R S} \sum_{p \in P_{n}^{r s}} \sum_{k \in K_{n}} \tilde{c}_{p}^{r s}\left(k, f_{n}^{*}\right)\left[f_{p}^{r s}(k)-f_{p}^{r s *}(k)\right] \geq 0 \forall f_{n} \in \Omega_{n} \\
& \Omega_{n}=\left\{f_{n} \mid f_{n} \geq 0, \sum_{p \in P_{n}^{r s}} \sum_{k \in K_{n}} \Delta_{n} \cdot f_{p}^{r s}(k)=Q^{r s}, \forall r s \in R S\right\}
\end{aligned}
$$

where $P_{n}^{r s}, K_{n}$ and $\Delta_{n}$ are fixed.

The proportional swap system proposed by Guo et al. (2017) for the bottleneck problem towards BR-UE can be extended to solve $\operatorname{VI}\left(f_{n}, \Omega_{n}\right)$ by the following formulas

$$
\begin{aligned}
f_{p n}^{r s(\tau+1)}(k)= & F_{p}^{r s}\left(f_{n}^{\tau}\right)=f_{p n}^{r s(\tau)}(k)+\eta \Gamma_{p n}^{r s}\left(k, f_{n}^{\tau}\right) \\
\Gamma_{p n}^{r s}\left(k, f_{n}^{\tau}\right)= & \sum_{p^{\prime} \in P_{n}^{r s}} \sum_{k^{\prime} \in K_{n}}\left(f_{p^{\prime} n}^{r s(\tau)}\left(k^{\prime}\right)\left[\tilde{c}_{p^{\prime} n}^{r s(\tau)}\left(k^{\prime}, f_{n}^{\tau}\right)-\tilde{c}_{p n}^{r s(\tau)}\left(k, f_{n}^{\tau}\right)\right]_{+}\right. \\
& \left.-f_{p n}^{r s(\tau)}(k)\left[\tilde{c}_{p n}^{r s(\tau)}\left(k, f_{n}^{\tau}\right)-\tilde{c}_{p^{\prime} n}^{r s(\tau)}\left(k^{\prime}, f_{n}^{\tau}\right)\right]_{+}\right)
\end{aligned}
$$

where a mapping $[\cdot]_{+}=\max \{\cdot, 0\}$ is used.

The continuity of $\tilde{c}_{p}^{r s}(k, f)$ makes $F_{p}^{r s}\left(f_{n}^{\tau}\right)$ continuous on $\Omega_{n}$. Moreover, $\Omega_{n}$ is nonempty, compact and convex. According to Guo et al. (2017), the proportional swap system has at least one stationary point, which is a BR-DUE state. The equivalence between a stationary point and a BR-DUE state can be derived directly from the equivalence theorems of Smith (1984) and Guo et al. (2017). Under the monotonicity assumption of the path disutility, Mounce (2007) and Mounce and Carey (2011) proved that the dynamical proportional swap system is convergent to an equilibrium.

Second, the CG algorithm can find the optimal solution to the $\operatorname{VI}(f, \Omega)$ problem through path extensions. Ignorance of the time dimension, the difference between the sub-problem Eqs. (C.1)-(C.2) and the original VI(f, $\Omega)$ problem Eqs. (7)-(9) lies in the path set. The number of paths of the original problem may be very large especially for large networks, but only a few paths have positive flows. The CG algorithm proposed by Leventhal et al. (1973) is able to find paths with positive flows as stated in Section 2.4. When no new path is found at iteration $n$, the rest any path $p \in P^{r s} \backslash P_{n}^{r s}$ has larger disutility and flows only concentrate on those identified paths. Therefore, the solution to the sub-problem Eqs. (C.1) and (C.2) is an equilibrium state of the BR-DUE problem.

According to Theorem 1, the above complete the proof of Corollary 1.

\section{References}

Bressan, A., Han, K., 2013. Existence of optima and equilibria for traffic flow on networks. Netw. Heterog. Media 8 (3), $627-648$.

Carey, M., Ge, Y.E., 2012. Comparison of methods for path flow reassignment for dynamic user equilibrium. Netw. Spat. Econ. 12 (3), 337-376.

Chen, A., Lo, H.K., Yang, H., 2001. A self-adaptive projection and contraction algorithm for the traffic assignment problem with path-specific costs. Eur. J. Oper. Res. 135 (1), 27-41.

Chen, B.Y., Lam, W.H.K., Sumalee, A., Shao, H., 2011. An efficient solution algorithm for solving multi-class reliability-based traffic assignment problem. Math. Comput. Model. 54 (5), 1428-1439.

Colak, S., Lima, A., González, M.C., 2016. Understanding congested travel in urban areas. Nat. Commun. 7, 1-8.

Daganzo, C.F., 1995. The cell transmission model, part II: network traffic. Transp. Res. Part B Methodol. 29 (2), $79-93$.

Dean, B.C., 2004. Shortest paths in FIFO time-dependent networks. Massachusetts Institute of Technology, pp. 1-13.

Di Lorenzo, D., Galligari, A., Sciandrone, M., 2014. A convergent and efficient decomposition method for the traffic assignment problem. Comput. Optim. Appl. 60 (1), 151-170.

Di, X., Liu, H.X., 2016. Boundedly rational route choice behavior: a review of models and methodologies. Transp. Res. Part B Methodol. 85, 142-179.

Fosgerau, Mogens, Karlstrom, A., 2010. The value of reliability. Transp. Res. Part B Methodol. 44 (1), 38-49.

Galligari, A., Sciandrone, M., 2017. A convergent and fast path equilibration algorithm for the traffic assignment problem. Optim. Methods Softw. 6788, 1-18.

Guo, R., Yang, H., Huang, H.J., Li, X., 2017. Day-to-day departure time choice under bounded rationality in the bottleneck model. Transp. Res. Part B Methodol. 23, 551-570.

Guo, X., Liu, H.X., 2011. Bounded rationality and irreversible network change. Transp. Res. Part B Methodol. 45 (10), $1606-1618$.

Han, K., Friesz, T.L., Yao, T., 2013. Existence of simultaneous route and departure choice dynamic user equilibrium. Transp. Res. Part B Methodol. 53, 17-30.

Han, K., Szeto, W.Y., Friesz, T.L., 2015. Formulation, existence, and computation of boundedly rational dynamic user equilibrium with fixed or endogenous user tolerance. Transp. Res. Part B Methodol. 79, 16-49.

Han, L., Ukkusuri, S., Doan, K., 2011. Complementarity formulations for the cell transmission model based dynamic user equilibrium with departure time choice, elastic demand and user heterogeneity. Transp. Res. Part B Methodol. 45 (10), 1749-1767.

Huang, H.J., Lam, W.H.K., 2002. Modeling and solving the dynamic user equilibrium route and departure time choice problem in network with queues. Transp. Res. Part B Methodol. 36 (3), 253-273.

Leventhal, T., Nemhauser, G., Trotter Jr, L., 1973. A column generation algorithm for optimal traffic assignment. Transp. Sci. 7 (2), $168-176$.

Levin, M.W., Pool, M., Owens, T., Juri, N.R., Travis Waller, S., 2015. Improving the convergence of simulation-based dynamic traffic assignment methodologies. Netw. Spat. Econ. 15 (3), 655-676.

Li, Q., Liao, F., Timmermans, H.J.P., Huang, H.J., Zhou, J., 2018. Incorporating free-floating car-sharing into an activity-based dynamic user equilibrium model: A demand-side model. Transp. Res. Part B Methodol. 107, 102-123.

Liao, F., 2016. Modeling duration choice in space-time multi-state supernetworks for individual activity-travel scheduling. Transp. Res. Part C Emerg. Technol. $69,16-35$.

Liao, F., 2017. Joint travel problem in space-time multi-state supernetworks. Transportation doi:10.1007/s11116-017-9835-6.

Liao, F., Arentze, T.A., Timmermans, H.J.P., 2010. Supernetwork approach for multimodal and multiactivity travel planning. Transp. Res. Rec. J. Transp. Res. Board 2175 (1), 38-46. 
Liao, F., Arentze, T., Timmermans, H.J.P., 2011. Constructing personalized transportation networks in multi-state supernetworks: a heuristic approach. Int. J. Geogr. Inf. Sci. 25, 1885-1903.

Liao, F., Arentze, T.A., Timmermans, H.J.P., 2013. Incorporating space-time constraints and activity-travel time profiles in a multi-state supernetwork approach to individual activity-travel scheduling. Transp. Res. Part B Methodol. 55, 41-58.

Liu, P., Liao, F., Huang, H.J., Timmermans, H.J.P., 2015. Dynamic activity-travel assignment in multi-state supernetworks. Transp. Res. Part B Methodol. 81, 656-671.

Lo, H.K., Szeto, W.Y., 2002. A cell-based variational inequality formulation of the dynamic user optimal assignment problem. Transp. Res. Part B Methodol. 36 (5), 421-443.

Long, J., Huang, H.J., Gao, Z., 2013a. Discretised route travel time models based on cumulative flows. J. Adv. Transp. 47 (1), $105-125$.

Long, J., Huang, H.J., Gao, Z., Szeto, W.Y., 2013b. An intersection-movement-based dynamic user optimal route choice problem. Oper. Res. 61 (5), $1134-1147$.

Long, J., Szeto, W.Y., Gao, Z., Huang, H.J., Shi, Q., 2016. The nonlinear equation system approach to solving dynamic user optimal simultaneous route and departure time choice problems. Transp. Res. Part B Methodol. 83, 179-206.

Lu, C.C., Liu, J., Qu, Y., Peeta, S., Rouphail, N.M., Zhou, X., 2016. Eco-system optimal time-dependent flow assignment in a congested network. Transp. Res. Part B Methodol. 94, 217-239.

Lu, C.C., Mahmassani, H.S., Zhou, X., 2009. Equivalent gap function-based reformulation and solution algorithm for the dynamic user equilibrium problem. Transp. Res. Part B Methodol. 43 (3), 345-364.

Lu, C.C., Zhou, X., Zhang, K., 2013. Dynamic origin-destination demand flow estimation under congested traffic conditions. Transp. Res. Part C Emerg. Technol. 34, 16-37.

Mounce, R., 2007. Existence of equilibrium in a continuous dynamic queueing model for traffic networks. In: Proceedings of the Fourth IMA International Conference on Mathematics in TransportInstitute of Mathematics and Its Applications.

Mounce, R., Carey, M., 2015. On the convergence of the method of successive averages for calculating equilibrium in traffic networks. Transp. Sci. 49 (3), $535-542$.

Mounce, R., Carey, M., 2011. Route swapping in dynamic traffic networks. Transp. Res. Part B Methodol. 45 (1), $102-111$.

Nagurney, A., Zhang, D., 1997. Projected dynamical systems in the formulation, stability analysis, and computation of fixed-demand traffic network equilibria. Transp. Sci. 31 (2), 147-158.

Nie, X., Zhang, H.M., 2005. Delay-function-based link models: their properties and computational issues. Transp. Res. Part B Methodol. 39 (8), 729-751.

Panicucci, B., Pappalardo, M., Passacantando, M., 2007. A path-based double projection method for solving the asymmetric traffic network equilibrium problem. Optim. Lett. 1 (2), 171-185.

Rubio-Ardanaz, J.M., Wu, J.H., Florian, M., 2003. Two improved numerical algorithms for the continuous dynamic network loading problem. Transp. Res. Part B Methodol. 37 (2), 171-190.

Ryu, S., Chen, A., Choi, K., 2016. Solving the stochastic multi-class traffic assignment problem with asymmetric interactions, route overlapping, and vehicle restrictions. J. Adv. Transp. 50 (2), 512-525.

Shao, H., Lam, W.H.K., Sumalee, A., Chen, A., 2013. Journey time estimator for assessment of road network performance under demand uncertainty. Transp. Res. Part C Emerg. Technol. 35, 244-262.

Sheffi, Y., 1985. Urban Transportation Networks. Prentice-Hall, Englewood Cliffs, NJ.

Simon, H.A., 1957. Models of Man: Social and Rationa;. Mathematical Essays on Rational Human Behavior in a Social Setting. Wiley, New York.

Simon, H.A., 1955. A behavioural model of rational choice. Q. J. Econ. 69 (1), 99-118.

Smith, M.J., 1984. The stability of a dynamic model of traffic assignment-an application of a method of lyapunov. Transp. Sci. 18 (3), 245.

Smith, M.J., Wisten, M.B., 1995. A continuous day-to-day traffic assignment model and the existence of a continuous dynamic user equilibrium. Ann. Oper. Res. 60, 59-79.

Szeto, W., Lo, H.K., 2006. Dynamic traffic assignment: properties and extensions. Transportmetrica 2 (1), 31-52.

Szeto, W.Y., Jiang, Y., Wong, K.I., Solayappan, M., 2013. Reliability-based stochastic transit assignment with capacity constraints: Formulation and solution method. Transp. Res. Part C Emerg. Technol. 35, 286-304.

Szeto, W.Y., Lo, H.K., 2004. A cell-based simultaneous route and departure time choice model with elastic demand. Transp. Res. Part B Methodol. 38 (7), 593-612.

Tong, C.O., Wong, S.C., 2000. A predictive dynamic traffic assignment model in congested capacity-constrained road networks. Transp. Res. Part B Methodol. $34(8), 625-644$

Wardrop, J.G., 1952. Some theoretical aspects of road traffic research. Proc. Inst. Civ. Eng. 1, 325-378.

Wu, J., Sun, H., Wang, D.Z.W., Zhong, M., Han, L., Gao, Z., 2013. Bounded-rationality based day-to-day evolution model for travel behavior analysis of urban railway network. Transp. Res. Part C Emerg. Technol. 31, 73-82.

Yang, H., Bell, M.G.H., 1998. Models and algorithms for road network design: a review and some new developments. Transp. Rev. 18 (3), $257-278$.

Yperman, I., 2007. The link transmission model for dynamic network loading. Katholieke Universiteit Leuven, Leuven, Belgium.

Zhang, K., Mahmassani, H.S., Lu, C.C., 2013. Dynamic pricing, heterogeneous users and perception error: probit-based bi-criterion dynamic stochastic user equilibrium assignment. Transp. Res. Part C Emerg. Technol. 27, 189-204.

Zhou, X., Mahmassani, H.S., Zhang, K., 2008. Dynamic micro-assignment modeling approach for integrated multimodal urban corridor management. Transp. Res. Part C Emerg. Technol. 16 (2), 167-186.

Zhou, X., Taylor, J., 2014. DTAlite: a queue-based mesoscopic traffic simulator for fast model evaluation and calibration. Cogent Eng. 1 (1), 1-19. 